\title{
Vertex operator representations of quantum tori at roots of unity ${ }^{1}$
}

\author{
Y. Billig \& K. Zhao \\ Department of Mathematics and Statistics \\ Carleton University \\ Ottawa, Ontario, K1S 5B6 Canada \\ Email: billig@math.carleton.ca \\ and \\ Institute of Mathematics \\ Academy of Mathematics and System Sciences \\ Chinese Academy of Sciences \\ Beijing 100080, P. R. China \\ Email: kzhao@mail2.math.ac.cn
}

ABSTRACT. As Lie algebra, we add the center $c_{1}$ (and the outer derivation $d_{1}$ ) to the quantum torus $\mathbb{C}_{q}$ to give the extended torus Lie algebra $\widehat{\mathbb{C}}_{q}$ (and $\widetilde{\mathbb{C}}_{q}$ respectively). Before the present paper, only some level 1 vertex operator representations for some $\widehat{\mathbb{C}}_{q}$ (and $\widetilde{\mathbb{C}}_{q}$ ) were constructed. In this paper, we first give vertex operator representations for $\mathrm{gl}_{I \times \infty}(\mathbb{C})$ where $I$ is an arbitrary index set. By embedding some $\widehat{\mathbb{C}}_{q}$ into $\mathrm{gl}_{I \times \infty}(\mathbb{C})$, we obtain a series of higher level vertex operator representations for $\widehat{\mathbb{C}_{q}}$ and $\widetilde{\mathbb{C}_{q}}$. Most of these vertex operator representations yield irreducible highest weight modules over these $\widetilde{\mathbb{C}_{q}}$. Also their character formulas follow directly.

\section{$\S 1$. Introduction}

Let $q=\left(q_{i, j}\right)_{i, j=0}^{n}$ be an $(n+1) \times(n+1)$ matrix over the complex number field $\mathbb{C}$ satisfying

$$
q_{i, i}=1, \quad q_{i, j}=q_{j, i}^{-1}
$$

where $n$ is a positive integer. The q-quantum torus $\mathbb{C}_{q}$ which was studied in $[\mathrm{MP}]$ is the unital associative algebra over $\mathbb{C}$ generated by $t_{0}^{ \pm 1}, \ldots, t_{n}^{ \pm 1}$ and subject to the defining relations

$$
t_{i} t_{j}=q_{i, j} t_{j} t_{i}
$$

\footnotetext{
${ }^{1}$ AMS Subject Classification: 17B20, 17B65, 17B67, 17B68, 16A40, 16A68.

This work is supported by NSERC, Hundred Talents Program of Chinese Academy of Sciences and by NSF of China.
} 
For any $a \in \mathbb{Z}^{n+1}$, we always write $a=\left(a_{0}, \ldots, a_{n}\right)$, and denote $t^{a}=t_{0}^{a_{0}} \ldots t_{n}^{a_{n}}$. For any $a, b \in \mathbb{Z}^{n+1}$, we define the functions $\sigma(a, b)$ and $f(a, b)$ by

$$
t^{a} t^{b}=\sigma(a, b) t^{a+b}, t^{a} t^{b}=f(a, b) t^{b} t^{a}
$$

We also define $|\alpha|=\alpha_{0}+\alpha_{1}+\ldots+\alpha_{n}$. Then

$$
\sigma(a, b)=\prod_{0 \leq i<j \leq n} q_{j, i}^{a_{j} b_{i}}, \quad f(a, b)=\prod_{i, j=0}^{n} q_{j, i}^{a_{j} b_{i}}
$$

and $f(a, b)=\sigma(a, b) \sigma(b, a)^{-1}$. We define $\operatorname{rad} f=\left\{a \in \mathbb{Z}^{n} \mid f\left(a, \mathbb{Z}^{n}\right)=1\right\}$ and the Kronecker delta

$$
\delta_{\alpha, \operatorname{rad} f}= \begin{cases}1, & \text { if } \alpha \in \operatorname{rad} f \\ 0, & \text { otherwise }\end{cases}
$$

For properties of $\mathbb{C}_{q}, f, \sigma$, please refer to $[\mathrm{BGK}]$ or $[\mathrm{Z}]$.

In most part of this paper we assume that

$$
q_{i, j}=1, \quad \forall 1 \leq i, j \leq n
$$

Note that (1.5) implies $\sigma\left(\alpha, \mathbb{Z}^{n+1}\right)=1$ for all $\alpha \in \operatorname{rad} f$. Under this assumption we have the 1-dimensional central extension $\hat{\mathbb{C}}_{q}=\mathbb{C}_{q} \oplus \mathbb{C} c$ of $\mathbb{C}_{q}$ with

$$
\left[t^{\alpha}, t^{\beta}\right]=t^{\alpha} t^{\beta}-t^{\beta} t^{\alpha}+\delta_{\alpha_{0},-\beta_{0}} \delta_{\alpha+\beta, \operatorname{rad}_{f}} \sigma(\alpha, \beta) \alpha_{0} c, \quad \forall \alpha, \beta \in \mathbb{Z}^{n+1} .
$$

We extend $\hat{\mathbb{C}}_{q}$ by a derivation $d_{0}$ with

$$
\left[d_{0}, t^{\alpha}\right]=\alpha_{0} t^{\alpha}, \quad \forall \alpha \in \mathbb{Z}^{n+1}
$$

to give the Lie algebra $\tilde{\mathbb{C}}_{q}=\hat{\mathbb{C}}_{q} \oplus \mathbb{C} d_{0}$. For any nonnegative integer $m$, and $l \in\{1,2, \ldots, n\}$, we modify (1.6) into

$$
\left[t^{\alpha}, t^{\beta}\right]=t^{\alpha} t^{\beta}-t^{\beta} t^{\alpha}+\delta_{\alpha_{0},-\beta_{0}} \delta_{\alpha+\beta, \operatorname{rad} f} \delta_{-\bar{\beta}_{l}, \bar{\alpha}_{l}} \sigma(\alpha, \beta) \alpha_{0} c, \quad \forall \alpha, \beta \in \mathbb{Z}^{n+1},
$$

where $\bar{\beta}_{l}, \bar{\alpha}_{l} \in \mathbb{Z}_{m}$, to get Lie algebras $\hat{\mathbb{C}}_{q}^{(l)}(m)$ and $\tilde{\mathbb{C}}_{q}^{(l)}(m)$ correspondingly. In some cases, $\tilde{\mathbb{C}}_{q}^{(l)}(m) \simeq \tilde{\mathbb{C}}_{q}^{(l)}\left(m^{\prime}\right)$ for some different $m$ and $m^{\prime}$. For example, if $n=1$ and $q_{1,0}^{k} \neq 1$ for all $k \in \mathbb{N}$, then $\tilde{\mathbb{C}}_{q}^{(1)}(m) \simeq \tilde{\mathbb{C}}_{q}$ for all $m \in \mathbb{Z}_{+}$. We know that $\hat{\mathbb{C}}_{q}, \tilde{\mathbb{C}}_{q}, \tilde{\mathbb{C}}_{q}^{(l)}(m)$ have a $\mathbb{Z}$-gradation with respect to $d_{0}$ :

$$
\tilde{\mathbb{C}}_{q}=\tilde{\mathbb{C}}_{q}^{(l)}(m)=\oplus_{k \in Z^{L}}
$$


where $L_{k}=\oplus_{\alpha \in \mathbb{Z}^{n}} \mathbb{C} t_{0}^{k} t_{1}^{\alpha_{1}} \ldots t_{n}^{\alpha_{n}} \oplus \delta_{k, 0}\left(\oplus \mathbb{C} c \oplus \mathbb{C} d_{0}\right)$. For a $\mathbb{Z}$-graded module $V=\oplus_{i \in \mathbb{Z}} V_{k}$, its character is defined as

$$
\operatorname{ch} V=\sum_{k \in \mathbb{Z}}\left(\operatorname{dim} V_{k}\right) x^{-k}
$$

In [BGT, BS, G1, G2, GL], level 1 vertex operator representations of the Lie algebras $\tilde{\mathbb{C}}_{q}$ were constructed, where they assumed $q^{\alpha}=q_{1,0}^{\alpha_{1}} \ldots q_{n, 0}^{\alpha_{n}} \neq 1$ if $\alpha \neq 0$. In this paper, we give not only higher level vertex operator representations of these Lie algebras $\tilde{\mathbb{C}}_{q}$ (Theorems $2.2,3.4$ ), but also construct higher level vertex operator representations of the Lie algebras $\tilde{\mathbb{C}}_{q}$ with $q_{1,2}$ being root of unity and all other $q_{i, j}$ being 1 for $i, j \in 1,2, \ldots, n$ (Theorems 4.1 and 4.5). Most of these vertex operator representations constructed are irreducible (Theorems 2.2, 3.6, 4.4, 4.6). The character formulas of these representations follow easily. All irreducible vertex operator representations constructed in this paper are highest weight modules (Theorem 5.1), thus the isomorphisms between these modules are clear.

\section{$\S 2$. Principal vertex operator representations for $\tilde{\mathbb{C}}_{q}$}

In this section we shall embed some $\hat{\mathbb{C}}_{q}$ in $\mathrm{gl}_{\infty}(\mathbb{C})$ to give principal vertex operator representations for $\hat{\mathbb{C}}_{q}$ and $\tilde{\mathbb{C}}_{q}$. Let us first recall a vertex operator representation theorem from [DJKM]. Let

$$
\overline{\mathcal{A}}_{\infty}=\left\{\left(a_{i, j}\right)_{i, j \in \mathbb{Z}} \mid a_{i, j} \in \mathbb{C}, \text { and } a_{i, j}=0 \text { if }|i-j| \gg 0\right\}
$$

be the infinite matrix Lie algebra. We use $E_{i, j}$ to denote the matrix unit, i.e., the matrix with 1 in $(\mathrm{i}, \mathrm{j})$-entry, and 0 elsewhere. Let $\mathcal{A}_{\infty}=\overline{\mathcal{A}}_{\infty}+\mathbb{C} c$ be the 1 -dimensional central extension with

$$
[X, Y]=X Y-Y X+\phi(X, Y) c, \quad \forall X, Y \in \overline{\mathcal{A}}_{\infty}
$$

where the 2-cocycle $\phi$ is given by

$$
\phi\left(E_{i, j}, E_{k, l}\right)=\left\{\begin{array}{l}
\delta_{i, l} \delta_{j, k}, \quad \text { if } i \leq 0, j \geq 1 \\
-\delta_{i, l} \delta_{j, k}, \text { if } j \leq 0, i \geq 1 \\
0, \text { otherwise }
\end{array}\right.
$$

Theorem 2.1 [DJMK] For any $m \in \mathbb{Z}$, the Lie algebra $\mathcal{A}_{\infty}$ has an irreducible vertex operator representation $R_{m}$ on the Fock space $B=\mathbb{C}\left[x_{1}, x_{2}, x_{3}, \ldots\right]$ so that

$$
R_{m}\left(\sum_{i, j \in \mathbb{Z}} u^{i} v^{-j} E_{i, j}\right)
$$




$$
\begin{gathered}
=\frac{1}{1-v / u}\left[(u / v)^{m} \exp \left(\sum_{j \in \mathbb{N}}\left(u^{j}-v^{j}\right) x_{j}\right) \exp \left(-\sum_{j \in \mathbb{N}} \frac{\left(u^{-j}-v^{-j}\right)}{j} \frac{\partial}{\partial x_{j}}\right)-1\right], \\
R_{m}(c)=1, \quad R_{m}\left(\sum_{j \in \mathbb{Z}} E_{j, i+j}\right)=\left\{\begin{array}{l}
\frac{\partial}{\partial x_{i}}, \text { if } i>0, \\
-i x_{-i}, \text { if } i<0, \\
m, \text { if } i=0,
\end{array}\right.
\end{gathered}
$$

where $\mathbb{N}$ is the set of natural numbers.

Part of the following theorem $\left(q^{\alpha}=q_{1,0}^{\alpha_{1}} \ldots q_{n, 0}^{\alpha_{n}} \neq 1\right.$ if $\left.\alpha \neq 0\right)$ was proved in [G1] and [GL]. The proof here is slightly different from that in [GL].

Theorem 2.2([GL]) Suppose $q=\left(q_{i, j}\right)_{i, j=0}^{n}$ satisfies (1.1) and (1.5). Then $\hat{\mathbb{C}}_{q}$ has an irreducible vertex operator representation $R_{m}$ for any $m \in \mathbb{Z}$ on the Fock space $B=$ $\mathbb{C}\left[x_{1}, x_{2}, x_{3}, \ldots\right]$ so that for any $\alpha=\left(\alpha_{1}, \ldots, \alpha_{n}\right) \in \mathbb{Z}^{n}$, we have

$$
\begin{gathered}
R_{m}\left(\sum_{j \in \mathbb{Z}} t_{0}^{j} t^{\alpha} z^{-j}\right)=\frac{q^{-m \alpha}}{1-q^{\alpha}} \exp \left(\sum_{j \in \mathbb{N}}\left(1-q^{j \alpha}\right) x_{j} z^{j}\right) \exp \left(-\sum_{j \in \mathbb{N}} \frac{1-q^{-j \alpha}}{j} \frac{\partial}{\partial x_{j}} z^{-j}\right), \text { if } q^{\alpha} \neq 1, \\
R_{m}(c)=1, \quad R_{m}\left(t_{0}^{i} t^{\alpha}\right)=\left\{\begin{array}{l}
\frac{\partial}{\partial x^{\prime}}, \text { if } i>0, q^{\alpha}=1, \\
-i x_{-i}, \text { if } i<0, q^{\alpha}=1, \\
m, \text { if } i=0, q^{\alpha}=1,
\end{array}\right.
\end{gathered}
$$

where $t^{\alpha}=t_{1}^{\alpha_{1}} \ldots t_{n}^{\alpha_{n}}, q^{\alpha}=q_{1,0}^{\alpha_{1}} \ldots q_{n, 0}^{\alpha_{n}}$. In (2.2) $q^{-m \alpha}$ can be replaced by any multiplicative function $\gamma(\alpha) \in \mathbb{C}^{*}$. If we define

$$
R_{m}\left(d_{0}\right)\left(x_{1}^{k_{1}} \ldots x_{l}^{k_{l}}\right)=-\left(k_{1}+2 k_{2}+\ldots+l k_{l}\right)\left(x_{1}^{k_{1}} \ldots x_{l}^{k_{l}}\right),
$$

then $B$ becomes a $\tilde{\mathbb{C}}_{q}$-module with character formula

$$
\operatorname{ch}(B)=\frac{1}{\varphi\left(x^{-1}\right)}=\frac{1}{\prod_{i \in \mathbb{N}}\left(1-x^{-i}\right)} .
$$

Proof. It is straightforward to verify that the following linear map is a Lie algebra homomorphism:

$$
\begin{gathered}
\eta: \hat{\mathbb{C}}_{q} \rightarrow \mathcal{A}_{\infty}, \\
t_{0}^{i} t^{\alpha} \rightarrow \sum_{j \in \mathbb{Z}} q^{-j \alpha} E_{j-i, j}+\delta_{i, 0}\left(1-\delta_{q^{\alpha}, 1}\right) \frac{c}{1-q^{\alpha}}, \quad \forall(i, \alpha) \in \mathbb{Z}^{n+1}, \\
c \rightarrow c .
\end{gathered}
$$


Since $R_{m}$ is a product of $R_{0}$ and a Lie automorphism, it suffices to show the theorem for only $R_{0}$. Letting $u=z, v=q^{\alpha} z$ in (2.1) we deduce that (for $q^{\alpha} \neq 0$ and $R_{0}$ )

$$
\begin{gathered}
\frac{1}{1-q^{\alpha}} \exp \left(\sum_{j \in \mathbb{N}}\left(1-q^{j \alpha}\right) x_{j} z^{j}\right) \exp \left(-\sum_{j \in \mathbb{N}} \frac{\left(1-q^{-j \alpha}\right)}{j} \frac{\partial}{\partial x_{j}} z^{-j}\right) \\
=R_{0}\left(\sum_{i, j \in \mathbb{Z}} q^{-j \alpha} z^{i-j} E_{i, j}\right)+\frac{1}{1-q^{\alpha}}=R_{0}\left(\sum_{l, j \in \mathbb{Z}} q^{-j \alpha} z^{-l} E_{j-l, j}\right)+\frac{1}{1-q^{\alpha}} \\
=R_{0}\left(\sum_{l \in \mathbb{Z}}\left(\sum_{j \in \mathbb{Z}} q^{-j \alpha} E_{j-l, j}+\delta_{l, 0} \frac{c}{1-q^{\alpha}}\right) z^{-l}\right) \\
=R_{0}\left(\sum_{l \in \mathbb{Z}} \eta\left(t_{0}^{l} t^{\alpha}\right) z^{-l}\right) .
\end{gathered}
$$

The other parts of the theorem are quite clear.

Remark 2.3. The embedding (2.4) without center can be regarded as the following string

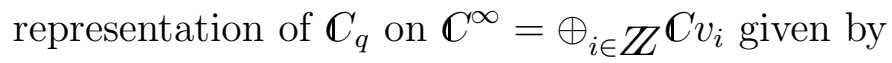

$$
\left(t_{0}^{k} t^{\alpha}\right) v_{i}=q^{-i \alpha} v_{i-k}, \quad \forall i, k \in \mathbb{Z}, \alpha \in \mathbb{Z}^{n}
$$

When $G_{q}=\mathbb{Z}^{n} \oplus \mathbb{Z}_{m}$, Theorem 2.2 is one of the main theorems in [G1], although they are slightly different in appearance.

Remark 2.4. If $\mathrm{n}=1$ and $q_{1,0}$ is a root of unity of order $r$, then representation $R_{0}$ is the level 1 representation of the affine Lie algebra $\hat{g l}{ }_{r}$.

\section{$\S 3$. Homogeneous vertex operator representations for $\hat{\mathbb{C}}_{q}$ and $\tilde{\mathbb{C}}_{q}$}

In this section we shall first construct a vertex operator representation for $\mathrm{gl}_{I \times \infty}(\mathbb{C})$ where $I$ is an index set, then embed $\hat{\mathbb{C}}_{q}$ in $\operatorname{gl}_{I \times \infty}(\mathbb{C})$ to give a series of higher level vertex operator representations for $\hat{\mathbb{C}}_{q}$ and $\tilde{\mathbb{C}}_{q}$ satisfying (1.5).

Let

$$
\begin{aligned}
& \overline{\mathcal{A}}_{I \times \infty}=\left\{\left(a_{i, j}^{k, l}\right) \mid i, j \in I, k, l \in \mathbb{Z}, a_{i, j}^{k, l} \in \mathbb{C}, \text { and } a_{i, j}^{k, l}=0\right. \\
& \left.\quad \text { if }|k-l| \gg 0, \text { and for fixed }(k, l) a_{i, j}^{k, l} \neq 0 \text { only for finitely many }(i, j)\right\}
\end{aligned}
$$

be the infinite matrix Lie algebra. Note that we allow $I$ to be an infinite set. We use $E_{i, j}^{k, l}$ to denote the matrix unit, i.e., the matrix with 1 in $(\mathrm{k}, \mathrm{l})$-(i,j)-entry, and 0 elsewhere. The products are given by

$$
E_{i, j}^{k, l} E_{i^{\prime}, j^{\prime}}^{k^{\prime}, l^{\prime}}=\delta_{j, i^{\prime}} \delta_{l, k^{\prime}} E_{i, j^{\prime}}^{k, l^{\prime}}
$$




$$
\left[E_{i, j}^{k, l}, E_{i^{\prime}, j^{\prime}}^{k^{\prime}, l^{\prime}}\right]=\delta_{j, i^{\prime}} \delta_{l, k^{\prime}} E_{i, j^{\prime}}^{k, l^{\prime}}-\delta_{j^{\prime}, i} \delta_{l^{\prime}, k} E_{i^{\prime}, j}^{k^{\prime}, l} .
$$

Let $\mathcal{A}_{I \times \infty}=\overline{\mathcal{A}}_{I \times \infty}+\mathbb{C} c$ be the 1-dimensional central extension with

$$
[X, Y]=X Y-Y X+\phi(X, Y) c
$$

where the 2-cocycle $\phi$ is given by

$$
\phi\left(E_{i, j}^{k, l}, E_{i^{\prime}, j^{\prime}}^{k^{\prime}, l^{\prime}}\right)=\left\{\begin{array}{l}
\delta_{i, j^{\prime}} \delta_{l^{\prime}, k} \delta_{i^{\prime}, j} \delta_{l, k^{\prime}}, \text { if } k \leq 0, l \geq 1 \\
-\delta_{i, j^{\prime}} \delta_{l^{\prime}, k} \delta_{i^{\prime}, j} \delta_{l, k^{\prime}}, \quad \text { if } l \leq 0, k \geq 1, \\
0, \quad \text { otherwise. }
\end{array}\right.
$$

Let $P_{I}=\oplus_{i \in I} \mathbb{Z} \epsilon_{i}$ be a free abelian additive group with a symmetric $\mathbb{Z}$-bilinear form $(\cdot \mid \cdot)$ defined by $\left(\epsilon_{i} \mid \epsilon_{j}\right)=\delta_{i, j}$ for all $i, j \in I$. Let

$$
Q_{I}=\oplus_{i, j \in I} \mathbb{Z}\left(\epsilon_{i}-\epsilon_{j}\right)
$$

be a subgroup of $P_{I}$. We see that

$$
(\alpha \mid \beta) \in 2 \mathbb{Z}, \forall \alpha, \beta \in \mathbb{Q}_{I}
$$

Let $\varepsilon: Q_{I} \times Q_{I} \rightarrow\{ \pm 1\}$ be a bimultiplicative function in the sense that

$$
\varepsilon(\alpha+\beta, \gamma)=\varepsilon(\alpha, \gamma) \varepsilon(\beta, \gamma), \quad \varepsilon(\alpha, \beta+\gamma)=\varepsilon(\alpha, \beta) \varepsilon(\alpha, \gamma)
$$

for all $\alpha, \beta, \gamma \in Q_{I}$, and with the property

$$
\varepsilon(\alpha, \alpha)=(-1)^{(\alpha \mid \alpha) / 2}, \quad \forall \alpha \in Q_{I} .
$$

It follows from (3.6) that

$$
\varepsilon(\alpha, \beta) \varepsilon(\beta, \alpha)=(-1)^{(\alpha \mid \beta)}, \quad \forall \alpha, \beta \in Q_{I} .
$$

Set $H_{I}=P_{I} \otimes_{\mathbb{Z}} \mathbb{C}$, and extend the $\mathbb{Z}$-bilinear form $(\cdot \mid \cdot)$ to get a symmetric $\mathbb{C}$-bilinear form $(\cdot \mid \cdot)$ on $H_{I}$. Let $\hat{H}_{I}=H_{I} \otimes \mathbb{C}\left[t, t^{-1}\right] \oplus \mathbb{C} c \oplus \mathbb{C} d_{0}$ be the Heisenberg Lie algebra with

$$
\begin{gathered}
{[\alpha(m), \beta(n)]=\delta_{m,-n} m(\alpha, \beta) c, \quad \forall \alpha, \beta \in H_{I}, m, n \in \mathbb{Z},} \\
{\left[d_{0}, c\right]=0, \quad\left[d_{0}, \alpha(m)\right]=m \alpha(m), \quad \forall \alpha \in H_{I}, m \in \mathbb{Z},}
\end{gathered}
$$

where $\alpha(n)=\alpha \otimes t^{n}$. Let $\hat{H}_{I}^{+}=\sum_{i>0} H_{I} \otimes t^{i}, \hat{H}_{I}^{-}=\sum_{i<0} H_{I} \otimes t^{i}$, then we have a subalgebra

$$
\mathcal{H}_{I}=\hat{H}_{I}^{+} \oplus \hat{H}_{I}^{-} \oplus \mathbb{C} c \oplus \mathbb{C} d_{0} .
$$


Let

$$
S\left(\hat{H}_{I}^{-}\right)=\mathbb{C}\left[\epsilon_{i}(-n) \mid i \in I, n \in \mathbb{N}\right] .
$$

Define the degree $\operatorname{deg}\left(\epsilon_{i_{1}}\left(-n_{1}\right) \epsilon_{i_{2}}\left(-n_{2}\right) \ldots \epsilon_{i_{r}}\left(-n_{r}\right)\right)$ to be

$$
\operatorname{deg}\left(\epsilon_{i_{1}}\left(-n_{1}\right) \epsilon_{i_{2}}\left(-n_{2}\right) \ldots \epsilon_{i_{r}}\left(-n_{r}\right)\right)=-\left(n_{1}+n_{2}+\ldots+n_{r}\right) .
$$

Then $\mathcal{H}_{I}$ has the natural representation on $S\left(\hat{H}_{I}^{-}\right)$in the sense that $\epsilon_{i}(n)$ acts as multiplication operator for $n<0, \epsilon_{i}(n)$ acts as differential operator $\frac{n \partial}{\partial \epsilon_{i}(-n)}$ for $n>0$, while $c$ acts as the identity, and $d_{0}$ acts as the degree operator.

Let

$$
\mathbb{C}\left[Q_{I}\right]=\oplus_{\alpha \in Q_{I}} \mathbb{C} e^{\alpha}
$$

be the group algebra of $Q_{I}$. For any $\beta \in Q_{I}$, define $e_{\beta} \in \operatorname{End} \mathbb{C}\left[Q_{I}\right]$ by

$$
e_{\beta} e^{\alpha}=\varepsilon(\beta, \alpha) e^{\alpha+\beta}, \quad \forall \alpha \in Q_{I}
$$

We see that

$$
e_{\alpha} e_{\beta}=\varepsilon(\alpha, \beta) e_{\alpha+\beta}, \quad \forall \alpha, \beta \in Q_{I} .
$$

For any $\beta \in H$, define $\beta(0) \in \operatorname{End} \mathbb{C}\left[Q_{I}\right]$ by

$$
\beta(0) e^{\alpha}=(\beta \mid \alpha) e^{\alpha}, \quad \forall \alpha \in Q_{I}
$$

It is clear that

$$
\left[\beta(0), e_{\alpha}\right]=(\beta \mid \alpha) e_{\alpha}, \quad \forall \alpha \in Q_{I}
$$

We define

$$
d_{0} e^{\alpha}=-\frac{(\alpha \mid \alpha)}{2} e^{\alpha}, \quad \forall \alpha \in Q_{I}
$$

Then

$$
\left[d_{0}, e_{\alpha}\right]=e_{\alpha}\left(-\alpha(0)-\frac{(\alpha \mid \alpha)}{2}\right), \quad \forall \alpha \in Q_{I}
$$

Set

$$
V_{Q_{I}}=S\left(H_{I}^{-}\right) \otimes_{\mathbb{C}} \mathbb{C}\left[Q_{I}\right]
$$

be the Fock space. We embed $\operatorname{End} S\left(H_{I}^{-}\right)$and End $\mathbb{C}\left[Q_{I}\right]$ (respectively $\left(\operatorname{End} S\left(H_{I}^{-}\right)\right)[[z]]$ and $\left(\right.$ End $\left.\left.\mathbb{C}\left[Q_{I}\right]\right)[[z]]\right)$ canonically into End $V_{Q_{I}}$ (respectively $\left.\left(\operatorname{End} V_{Q_{I}}\right)[[z]]\right)$, for instance,

$$
\beta(l)=\left\{\begin{array}{ll}
\beta(l) \otimes 1, & \text { if } l \neq 0, \\
1 \otimes \beta(l), & \text { if } l=0
\end{array}, \forall \beta \in H_{I}\right.
$$

We define the action of $d_{0}$ on $V_{Q_{I}}$ by

$$
d_{0}=d_{0} \otimes 1+1 \otimes d_{0} .
$$


Recall that $\left.z^{\alpha} \in \operatorname{End} \mathbb{C}\left[Q_{I}\right]\right)[[z]]$ is given by

$$
z^{\alpha} e^{\beta}=z^{(\alpha \mid \beta)} e^{\beta}, \quad \forall \alpha, \beta \in Q_{I}
$$

Thus we have

$$
\left[\alpha(0), z^{\beta}\right]=0, \quad z^{\alpha} e_{\beta}=e_{\beta} z^{\alpha+(\alpha \mid \beta)}, \quad \forall \alpha, \beta \in Q_{I}
$$

Similarly, for any nonzero complex number $a$, we have the evaluation $a^{\alpha}$ of $z^{\alpha}$ so that $a^{\alpha} \in$ $\operatorname{End}\left(\mathbb{C}\left[Q_{I}\right]\right)$.

Now we modify $\mathcal{A}_{I \times \infty}$ to give the Lie algebra $\mathcal{A}_{I \times \infty}(\varepsilon)$ as follows. As vector spaces, $\mathcal{A}_{I \times \infty}=\mathcal{A}_{I \times \infty}(\varepsilon)$. But the Lie bracket is given by

$$
\begin{array}{r}
{\left[\dot{E}_{i, j}^{k, l}, \dot{E}_{i^{\prime}, j^{\prime}}^{k^{\prime}, l^{\prime}}\right]=\varepsilon\left(\epsilon_{i}-\epsilon_{j}, \epsilon_{i^{\prime}}-\epsilon_{j^{\prime}}\right) \delta_{j, i^{\prime}} \delta_{l, k^{\prime}} \dot{E}_{i, j^{\prime}}^{k, l^{\prime}}-\varepsilon\left(\epsilon_{i^{\prime}}-\epsilon_{j^{\prime}}, \epsilon_{i}-\epsilon_{j}\right) \delta_{j^{\prime}, i} \delta_{l^{\prime}, k} \dot{E}_{i^{\prime}, j}^{k^{\prime}, l}} \\
+\varepsilon\left(\epsilon_{i}-\epsilon_{j}, \epsilon_{i^{\prime}}-\epsilon_{j^{\prime}}\right) \phi\left(E_{i, j}^{k, l}, E_{i^{\prime}, j^{\prime}}^{k^{\prime}, l^{\prime}}\right) c, \quad \forall, i, j, i^{\prime}, j^{\prime} \in I, \quad k, l, k^{\prime}, l^{\prime} \in \mathbb{Z} .
\end{array}
$$

Here we used $\dot{E}_{i, j}^{k, l}$ (which is viewed as matrix unit in $\mathcal{A}_{I \times \infty}(\varepsilon)$ ) to distinguish the matrix unit with the ordinary one $E_{i, j}^{k, l}$ which is viewed as matrix unit in $\left.\mathcal{A}_{I \times \infty}\right)$. We know that the Lie algebras $\mathcal{A}_{I \times \infty}$ and $\mathcal{A}_{I \times \infty}(\varepsilon)$ are isomorphic.

The following theorem is inspired by Theorem 2.1, [Theorem 1.2, TV], and the results in $[\mathrm{F}]$.

Theorem 3.1 The Lie algebra $\mathcal{A}_{I \times \infty}(\varepsilon)$ has an irreducible vertex operator representation $R_{m}$ for any $m \in \mathbb{Z}$ on the Fock space $V_{Q_{I}}$ given by

$$
\begin{gathered}
R_{m}\left(\sum_{k, l \in \mathbb{Z}} \dot{E}_{i, j}^{k, l} z_{1}^{k} z_{2}^{-l}\right)=-\frac{\delta_{i, j}}{\left(1-z_{2} / z_{1}\right)}+e_{\epsilon_{i}-\epsilon_{j}} z_{1}^{\epsilon_{i}+1+m-\delta_{i, j}} z_{2}^{-\epsilon_{j}-m}\left(1-z_{2} / z_{1}\right)^{-\delta_{i, j}} . \\
\cdot \exp \left(-\sum_{p \in-\mathbb{N}} \frac{z_{1}^{-p} \epsilon_{i}(p)-z_{2}^{-p} \epsilon_{j}(p)}{p}\right) \exp \left(-\sum_{p \in \mathbb{N}} \frac{z_{1}^{-p} \epsilon_{i}(p)-z_{2}^{-p} \epsilon_{j}(p)}{p}\right), \quad \forall i, j \in I, \\
R_{m}(c)=1, \quad R_{m}\left(\sum_{k \in \mathbb{Z}} \dot{E}_{i, i}^{k, k+l}\right)=\epsilon_{i}(l), \quad \forall l \in \mathbb{Z} .
\end{gathered}
$$

Proof. Since the proof of this theorem is quite standard (although onerous), instead of giving the detailed proof we shall only sketch the proof.

We can verify (via a lot of computations) that

$$
\left[R_{m}\left(\sum_{k, l \in \mathbb{Z}} \dot{E}_{i, j}^{k, l} z_{1}^{k} z_{2}^{-l}\right), R_{m}\left(\sum_{k, l \in \mathbb{Z}} \dot{E}_{j, j^{\prime}}^{k, l} z_{3}^{k} z_{4}^{-l}\right)\right]=R_{m}\left(\left[\sum_{k, l \in \mathbb{Z}} \dot{E}_{i, j}^{k, l} z_{1}^{k} z_{2}^{-l}, \sum_{k, l \in \mathbb{Z}} \dot{E}_{j, j^{\prime}}^{k, l} z_{3}^{k} z_{4}^{-l}\right]\right),
$$


for all cases: (1) $i \neq j \neq j^{\prime} \neq i$, (2) $i \neq j=j^{\prime}$, (3) $j^{\prime}=i \neq j$, (4) $i=j=j^{\prime}$ separately by using formula (3.37). So the first and the second formulas in (3.23) follow. The third formulas in (3.23) with $l \neq 0$ are clear from Theorem 2.1. The third formulas in (3.23) with $l=0$ follow from the facts that $\left[R_{m}\left(\sum_{k \in \mathbb{Z}} \dot{E}_{i, i}^{k, k}\right), R_{m}\left(\sum_{k \in \mathbb{Z}} \dot{E}_{i, i}^{k, k+l}\right)\right]=0$ for all $l \in \mathbb{Z}$, and

$$
\left.\left[R_{m}\left(\sum_{k \in \mathbb{Z}} \dot{E}_{i, i}^{k, k}\right), R_{m}\left(\sum_{k, l \in \mathbb{Z}} \dot{E}_{i^{\prime}, j^{\prime}}^{k, l} z_{1}^{k} z_{2}^{-l}\right)\right]=\left(\delta_{i, i^{\prime}}-\delta_{i, j^{\prime}}\right) R_{m}\left(\sum_{k, l \in \mathbb{Z}} \dot{E}_{i^{\prime}, j^{\prime}}^{k, l} z_{1}^{k} z_{2}^{-l}\right)\right]
$$

The irreducibility follows from the last equation of (3.23) and the action of $e_{\epsilon_{i}-\epsilon_{j}}$.

Theorem 3.2 Suppose q satisfies

$$
q_{i, 0}=q_{0, i}^{-1}, \quad q_{0,0}=q_{i, j}=q_{j, i}=1, \quad \forall i, j \in\{1,2, \ldots, n\} .
$$

Let $\widehat{g l}_{m}\left(\mathbb{C}_{q}, \varepsilon\right)=g l_{m}\left(\mathbb{C}_{q}\right) \oplus \mathbb{C} c$ be the 1 -dimensional central extension with

$$
\begin{aligned}
& {\left[\dot{E}_{i, j}\left(t^{\alpha}\right), \dot{E}_{i^{\prime}, j^{\prime}}\left(t^{\beta}\right)\right]=\delta_{j, i^{\prime}} \varepsilon\left(\epsilon_{i}-\epsilon_{j}, \epsilon_{i^{\prime}}-\epsilon_{j^{\prime}}\right) \dot{E}_{i, j^{\prime}}\left(t^{\alpha} t^{\beta}\right)-\delta_{j^{\prime}, i} \varepsilon\left(\epsilon_{i^{\prime}}-\epsilon_{j^{\prime}}, \epsilon_{i}-\epsilon_{j}\right) \dot{E}_{i^{\prime}, j}\left(t^{\beta} t^{\alpha}\right)} \\
& -\varepsilon\left(\epsilon_{i}-\epsilon_{j}, \epsilon_{i^{\prime}}-\epsilon_{j^{\prime}}\right) \delta_{j, i^{\prime}} \delta_{j^{\prime}, i} \delta_{\alpha_{0}+\beta_{0}, 0} \delta_{q^{-\beta}, q^{\alpha}} \sigma(\alpha, \beta) \alpha_{0} c, \quad \forall \alpha, \beta \in Z^{n+1}, i, j, i^{\prime}, j^{\prime} \in I .
\end{aligned}
$$

Then $\widehat{g l}_{m}\left(\mathbb{C}_{q}, \varepsilon\right)$ has an irreducible vertex operator representation $R$ on the Fock space $V_{Q_{I}}$ with $I=\{1,2, \ldots, m\}$ so that for any $\alpha=\left(\alpha_{1}, \ldots, \alpha_{n}\right) \in \mathbb{Z}^{n}$, any $i \neq j \in I$, we have

$$
\begin{gathered}
R\left(\sum_{p \in \mathbb{Z}} \dot{E}_{i, j}\left(t_{0}^{p} t^{\alpha}\right) z^{-p}\right)=e_{\epsilon_{i}-\epsilon_{j}} z^{\epsilon_{i}-\epsilon_{j}+1-\delta_{i, j}}\left(q^{\alpha}\right)^{-\epsilon_{j}}\left(1-q^{\alpha}\right)^{-\delta_{i, j}} \cdot \\
\cdot \exp \left(-\sum_{p \in-\mathbb{N}} \frac{\left(\epsilon_{i}(p)-q^{-p \alpha} \epsilon_{j}(p)\right)}{p} z^{-p}\right) \exp \left(-\sum_{p \in \mathbb{N}} \frac{\left(\epsilon_{i}(p)-q^{-p \alpha} \epsilon_{j}(p)\right)}{p} z^{-p}\right), \text { if } q^{\alpha} \neq 1 \\
R(c)=1, \quad R\left(\dot{E}_{i, i}\left(t_{0}^{l} t^{\alpha}\right)\right)=\epsilon_{i}(l), \text { if } \quad q^{\alpha}=1 .
\end{gathered}
$$

If we extend $\widehat{g l}_{m}\left(\mathbb{C}_{q}, \varepsilon\right)$ by $d_{0}$ to get the Lie algebra $\widetilde{g l}_{m}\left(\mathbb{C}_{q}, \varepsilon\right)$ with

$$
\left[d_{0}, \dot{E}_{i, j}\left(t^{\alpha}\right)\right]=\alpha_{0} \dot{E}_{i, j}\left(t^{\alpha}\right), \quad \forall \alpha \in \mathbb{Z}^{n+1},
$$

then $V_{Q_{I}}$ is a module over $\tilde{g l}\left(\mathbb{C}_{q}\right)$ via (3.19), and

$$
\operatorname{ch} V_{Q_{I}}=\left(\sum_{\alpha \in Q_{I}} x^{-(\alpha \mid \alpha) / 2}\right) \phi\left(x^{-1}\right)^{-m}
$$


Proof. It is straightforward to verify that the following linear map is a Lie algebra homomorphism:

$$
\begin{gathered}
\tau: \widehat{g l}_{m}\left(\mathbb{C}_{q}, \varepsilon\right) \rightarrow \mathcal{A}_{I \times \infty}(\varepsilon), \\
\dot{E}_{i, j}\left(t_{0}^{k} t^{\alpha}\right) \rightarrow \sum_{p \in \mathbb{Z}} q^{-p \alpha} \dot{E}_{i, j}^{p-k, p}+\frac{\delta_{k, 0} \delta_{i, j}\left(1-\delta_{1, q^{\alpha}}\right) c}{1-q^{\alpha}}, \forall(k, \alpha) \in \mathbb{Z}^{n+1}, i, j \in I \\
c \rightarrow c .
\end{gathered}
$$

Letting $z_{1}=z, z_{2}=q^{\alpha} z$ in $(2.23)$ we deduce that (for $q^{\alpha} \neq 1$ and $R_{0}$ )

$$
\begin{gathered}
e_{\epsilon_{i}-\epsilon_{j}} z^{\epsilon_{i}-\epsilon_{j}+1-\delta_{i, j}}\left(q^{\alpha}\right)^{-\epsilon_{j}}\left(1-q^{\alpha}\right)^{-\delta_{i, j}} . \\
\cdot \exp \left(-\sum_{p \in-\mathbb{N}} \frac{\left(\epsilon_{i}(p)-q^{-p \alpha} \epsilon_{j}(p)\right)}{p} z^{-p}\right) \exp \left(-\sum_{p \in \mathbb{I}} \frac{\left(\epsilon_{i}(p)-q^{-p \alpha} \epsilon_{j}(p)\right)}{p} z^{-p}\right) \\
=R_{0}\left(\sum_{k, l \in \mathbb{Z}} q^{-l \alpha} z^{k-l} \dot{E}_{i, j}^{k, l}\right)+\frac{\delta_{i, j}}{1-q^{\alpha}} \\
=R_{0}\left(\sum_{l, k \in \mathbb{Z}} q^{-l \alpha} z^{-k} \dot{E}_{i, j}^{l-k, l}\right)+\frac{\delta_{i, j}}{1-q^{\alpha}} \\
=R_{0}\left(\left(\sum_{k \in \mathbb{Z}}\left(\sum_{l \in \mathbb{Z}} q^{-l \alpha} \dot{E}_{i, j}^{l-k, l}\right)+\delta_{k, 0} \frac{\delta_{i, j} c}{1-q^{\alpha}}\right) z^{-k}\right) \\
=R_{0}\left(\sum_{k \in \mathbb{Z}} \tau\left(\dot{E}_{i, j}\left(t_{0}^{k} t^{\alpha}\right) z^{-k}\right)\right) .
\end{gathered}
$$

The other formulas of (3.25) are quite clear. To see that $V_{Q_{I}}$ is a module over $\tilde{g l}\left(\mathbb{C}_{q}\right)$, we need to show that

$$
\left[R\left(d_{0}\right), R\left(\sum_{p \in \mathbb{Z}} \dot{E}_{i, j}\left(t_{0}^{p} t^{\alpha}\right) z^{-p}\right)\right]=R\left(\sum_{p \in \mathbb{Z}} \dot{E}_{i, j}\left(t_{0}^{p} t^{\alpha}\right) p z^{-p}\right)=-\frac{z \partial}{\partial z} R\left(\sum_{p \in \mathbb{Z}} \dot{E}_{i, j}\left(t_{0}^{p} t^{\alpha}\right) z^{-p}\right)
$$

which can be verified by using (3.17) and (the action on $\mathbb{C}\left[Q_{I}\right]$ )

$$
z \frac{\partial}{\partial z} z^{\alpha}=\alpha(0) z^{\alpha}, \quad \forall \alpha \in Q_{I}
$$

Other parts of the theorem follows easily. Thus we complete the proof of this theorem.

Remark 3.3. The embedding (3.27) without center is inspired by the following string representation of $\operatorname{gl}_{m}\left(\mathbb{C}_{q}\right)$ on $\mathbb{C}^{m \times \infty}=\oplus_{i \in \mathbb{Z}^{m}, j \in \mathbb{Z}} \mathbb{C} v_{i, j}$ given by

$$
\left(E_{k, l}\left(t_{0}^{p} t^{\alpha}\right)\right) v_{i, j}=\delta_{i, l} q^{-j \alpha} v_{k, j-p}
$$


When $G_{q}=\mathbb{Z}^{2}$ or $\mathbb{Z}^{3}, I$ is finite, and $q_{1,0}^{i} q_{2,0}^{j} \neq 1$ for $(i, j) \neq(0,0)$, Theorem 3.2 is one of the main theorems in [G2], although they are slightly different in appearance.

Theorem 3.4 Let $q$ satisfy (3.24). For any positive integer $m$, let $\omega_{m}$ be the primitive root of unity of order $m, I=\mathbb{Z} / m \mathbb{Z}=\{1,2, \ldots, m\}$. Then $\hat{\mathbb{C}}_{q}^{(1)}(m)$ has a vertex operator representation $R_{m}$ on the Fock space $S\left(\hat{H}_{I}^{-}\right)$so that for any $\alpha=\left(\alpha_{1}, \ldots, \alpha_{n}\right) \in \mathbb{Z}^{n}$, we have

$$
\begin{gathered}
R_{m}\left(\sum_{p \in \mathbb{Z}}\left(t_{0}^{p} t^{\alpha}\right) z^{-p}\right)=\sum_{j=1}^{m} \frac{\omega_{m}^{-j \alpha_{1}}}{\left(1-q^{\alpha}\right)} \cdot \exp \left(-\sum_{p \in-\mathbb{N}} \frac{\left(1-q^{-p \alpha}\right) \epsilon_{j}(p)}{p} z^{-p}\right) . \\
\cdot \exp \left(-\sum_{p \in \mathbb{N}} \frac{\left(1-q^{-p \alpha}\right) \epsilon_{j}(p)}{p} z^{-p}\right), \text { if } q^{\alpha} \neq 1, \\
R_{m}(c)=m, \quad R_{m}\left(t_{0}^{l} t^{\alpha}\right)=\sum_{j=1}^{m} \omega_{m}^{-j \alpha_{1}} \epsilon_{j}(l), \text { if } q^{\alpha}=1 .
\end{gathered}
$$

Proof. It is straightforward to verify that the following linear map is a Lie algebra homomorphism:

$$
\begin{aligned}
\tau: \hat{\mathbb{C}}_{q}^{(1)}(m) \rightarrow \mathcal{A}_{I \times \infty}(\varepsilon) \\
t_{0}^{i} t^{\alpha} \rightarrow \sum_{j=1}^{m} \omega_{m}^{-j \alpha_{1}} \sum_{k \in \mathbb{Z}} q^{-k \alpha} \dot{E}_{j, j}^{k-i, k}+\frac{\delta_{i, 0} \delta_{\overline{\alpha_{1}, 0}}\left(1-\delta_{q^{\alpha}, 1}\right) m c}{1-q^{\alpha}} \\
c \rightarrow m c
\end{aligned}
$$

where $\overline{\alpha_{1}} \in \mathbb{Z}_{m}$. If $q^{\alpha} \neq 1$, using $R_{0}$ in Theorem 3.1 we deduce that

$$
\begin{gathered}
R_{m}\left(\sum_{k \in \mathbb{Z}}\left(t_{0}^{k} t^{\alpha}\right) z^{-k}\right):=R_{0}\left(\sum_{k \in \mathbb{Z}} \tau\left(t_{0}^{k} t^{\alpha}\right) z^{-k}\right) \\
=\sum_{k \in \mathbb{Z}} z^{-k} R_{0}\left(\sum_{j=1}^{m} \omega_{m}^{-j \alpha_{1}} \sum_{p \in \mathbb{Z}} q^{-p \alpha} \dot{E}_{j, j}^{p-k, p}+\frac{\delta_{k, 0} \delta_{\overline{\alpha_{1}}, 0} m c}{1-q^{\alpha}}\right) \\
=\sum_{j=1}^{m} \omega_{m}^{-j \alpha_{1}} \sum_{k \in \mathbb{Z}} z^{-k}\left(R_{0}\left(\sum_{p \in \mathbb{Z}} q^{-p \alpha} \dot{E}_{j, j}^{p-k, p}\right)+\frac{\delta_{k, 0}}{1-q^{\alpha}}\right) \\
\left(l e t t i n g \quad z_{1}=z, z_{2}=q^{\alpha} z i n(3.23) \quad w i t h i=j, \quad \operatorname{acting} \text { on } S\left(\hat{H}_{I}^{-}\right) \otimes 1\right) \\
=\sum_{j=1}^{m} \frac{\omega_{m}^{-j \alpha_{1}}}{\left(1-q^{\alpha}\right)} \cdot \exp \left(-\sum_{p \in-\mathbb{N}} \frac{\left(1-q^{-p \alpha}\right) \epsilon_{j}(p)}{p} z^{-p}\right) \exp \left(-\sum_{p \in \mathbb{N}} \frac{\left(1-q^{-p \alpha}\right) \epsilon_{j}(p)}{p} z^{-p}\right) .
\end{gathered}
$$


The other parts of the theorem are quite clear.

The following Lemma is very useful to further study on the structure of the module in Theorem 3.4.

Lemma 3.5. Let $\omega_{m}$ be given as in Theorem 3.4, $q_{1,0}, \ldots, q_{n, 0}$ be nonzero numbers, $W \subset V$ be vector spaces. Suppose the set $\left\{q^{\alpha}=q_{1,0}^{\alpha_{1}} \ldots q_{n, 0}^{\alpha_{n}} \mid \alpha \in \mathbb{Z}^{n}\right\}$ is infinite. Let $f_{j}\left(q^{\alpha}\right)=\sum_{i} q^{i \alpha} v_{i}^{(j)} \in$ $V$ for all $j=1,2 \ldots, m, \alpha \in \mathbb{Z}^{n}$. If $\sum_{j=1}^{m} \omega_{m}^{j \alpha_{1}} f_{j}\left(q^{\alpha}\right) \in W$ for all $\alpha \in \mathbb{Z}^{n}$, then $f_{j}\left(q^{\alpha}\right) \in W$ for all $j$ and all $\alpha \in \mathbb{Z}^{n}$.

Proof. We know that

$$
\sum_{i} q^{i \alpha} \sum_{j=1}^{m} \omega_{m}^{j \alpha_{1}} v_{i}^{(j)}=\sum_{j=1}^{m} \omega_{m}^{j \alpha_{1}} f_{j}\left(q^{\alpha}\right) \in W, \quad \forall \alpha \in \mathbb{Z}^{n} .
$$

By letting $\alpha_{1}=k m+i_{0}$ where $i_{0}=0,1, \ldots, m-1$ and $k \in \mathbb{Z}$, and changing other $\alpha_{i} \in \mathbb{Z}$ in (3.30), we obtain that

$$
\sum_{i} q^{i \alpha} \sum_{j=1}^{m} \omega_{m}^{j i_{0}} v_{i}^{(j)} \in W, \quad \forall \alpha \in \mathbb{Z}^{n} \text { with } \alpha_{1}=k m+i_{0}
$$

From the hypothesis we know that the set $\left\{q^{\alpha}=q_{1,0}^{\alpha_{1}} \ldots q_{n, 0}^{\alpha_{n}} \mid \alpha \in \mathbb{Z}^{n}\right.$ with $\left.a_{1}=i_{0} \bmod m\right\}$ is infinite. We deduce that

$$
\sum_{j=1}^{m} \omega_{m}^{j i_{0}} v_{i}^{(j)} \in W, \quad \forall i_{0} \in\{0,1, \ldots, m-1\}, i \in \mathbb{Z}
$$

It follows that $v_{i}^{(j)} \in W, \forall i, j \in \mathbb{Z}$. The lemma follows.

Theorem 3.6 $S\left(\hat{H}_{I}^{-}\right)$in Theorem 3.4 is a $\tilde{\mathbb{C}}_{q}^{(1)}(m)$-module. If, in addition to the assumptions in Theorem 3.4, we assume that the set $\left\{q^{\alpha}=q_{1,0}^{\alpha_{1} \ldots} q_{n, 0}^{\alpha_{n}} \mid \alpha \in \mathbb{Z}^{n}\right\}$ is infinite, or there exists $\alpha \in \mathbb{Z}^{n}$ such that $\left(\alpha_{1}, m\right)=1$ and $q^{\alpha}=1$, then $\tilde{\mathbb{C}}_{q}^{(1)}(m)$-module $S\left(\hat{H}_{I}^{-}\right)$is irreducible, and

$$
\operatorname{ch}\left(S\left(\hat{H}_{I}^{-}\right)\right)=\varphi\left(x^{-1}\right)^{-m}
$$

Proof. It is clear that $S\left(\hat{H}_{I}^{-}\right)$is a $\tilde{\mathbb{C}}_{q}^{(1)}(m)$-module. If there exists $\alpha \in \mathbb{Z}^{n}$ such that $\left(\alpha_{1}, m\right)=1$ and $q^{\alpha}=1$, from the third equation of (3.28) we see that

$$
\epsilon_{j}(l) \in R_{m}\left(\tilde{\mathbb{C}}_{q}^{(1)}(m)\right), \quad \forall j \in I, l \in \mathbb{Z} .
$$


Thus $\tilde{\mathbb{C}}_{q}^{(1)}(m)$-module $S\left(\hat{H}_{I}^{-}\right)$in Theorem 3.4 is irreducible. Next we suppose $\left\{q^{\alpha} \mid \alpha \in \mathbb{Z}^{n}\right\}$ is infinite. For $q^{\alpha} \neq 1$, let

$$
f_{j}\left(q^{\alpha}, z\right)=\frac{1}{\left(1-q^{\alpha}\right)} \exp \left(-\sum_{p \in-\mathbb{N}} \frac{\left(1-q^{-p \alpha}\right) \epsilon_{j}(p)}{p} z^{-p}\right) \exp \left(-\sum_{p \in \mathbb{N}} \frac{\left(1-q^{-p \alpha}\right) \epsilon_{j}(p)}{p} z^{-p}\right)
$$

For any $v \in S\left(\hat{H}_{I}^{-}\right)$, from $(3.28)$ we know that

$$
\sum_{j=1}^{m} \omega_{m}^{-j \alpha_{1}} f_{j}\left(q^{\alpha}, z\right) v \in\left(R_{m}\left(\widetilde{\mathbb{C}}_{q}^{(1)}(m)\right) v\right)\left[z, z^{-1}\right], \quad \forall \alpha \in \mathbb{Z}^{n} \text { with } q^{\alpha} \neq 1
$$

Using Lemma 3.5, we see that, for any $j$,

$$
f_{j}\left(q^{\alpha}, z\right) v \in\left(R_{m}\left(\widetilde{\mathbb{C}}_{q}^{(1)}(m)\right) v\right)\left[z, z^{-1}\right], \quad \forall v \in S\left(\hat{H}_{I}^{-}\right), \alpha \in \mathbb{Z}^{n} \text { with } q^{\alpha} \neq 1
$$

It is clear that, for any $j$,

$$
\begin{aligned}
{\left[f_{j}\left(q^{\alpha}, z_{1}\right), f_{j}\left(q^{-\alpha}, z_{2}\right)\right] v \in } & \left(R_{m}\left(U\left({\widetilde{\mathbb{C}_{q}}}^{(1)}(m)\right)\right) v\right)\left[z_{1}^{ \pm 1}, z_{2}^{ \pm 1}\right], \\
& \forall v \in S\left(\hat{H}_{I}^{-}\right), \alpha \in \mathbb{Z}^{n} \text { with } q^{\alpha} \neq 1 .
\end{aligned}
$$

By using the following well known formulas

$$
\begin{gathered}
f\left(z_{1}, z_{2}\right) D \delta\left(\frac{z_{2}}{q^{\alpha} z_{1}}\right)=f\left(z_{1}, q^{\alpha} z_{1}\right) D \delta\left(\frac{z_{2}}{q^{\alpha} z_{1}}\right)-q^{\alpha} z_{1}\left(\frac{\partial}{\partial z_{2}} f\left(z_{1}, z_{2}\right)\right) \delta\left(\frac{z_{2}}{q^{\alpha} z_{1}}\right), \\
f\left(z_{1}, z_{2}\right) \delta\left(\frac{z_{2}}{q^{\alpha} z_{1}}\right)=f\left(z_{1}, q^{\alpha} z_{1}\right) \delta\left(\frac{z_{2}}{q^{\alpha} z_{1}}\right)
\end{gathered}
$$

where

$$
\delta(z)=\sum_{p \in \mathbb{Z}} z^{p}, \quad(D \delta)(z)=\sum_{p \in \mathbb{Z}} p z^{p}
$$

it is standard to show that, if $q^{\alpha} \neq 1$, then

$$
\left[f_{j}\left(q^{\alpha}, z_{1}\right), f_{j}\left(q^{-\alpha}, z_{2}\right)\right]=\sum_{p, p^{\prime} \in \mathbb{Z}} q^{-p \alpha}\left(q^{\left(p+p^{\prime}\right) \alpha}-1\right) \epsilon_{j}\left(p+p^{\prime}\right) z_{1}^{-p} z_{2}^{-p^{\prime}}+(D \delta)\left(\frac{z_{2}}{q^{\alpha} z_{1}}\right) .
$$

Since $\left\{q^{\alpha} \mid \alpha \in \mathbb{Z}^{n}\right\}$ is infinite, we see that for any $i \neq 0$, there exists $\alpha \in \mathbb{Z}^{n}$ such that $q^{i \alpha} \neq 1$. Thus

$$
\epsilon_{j}(p) \in R_{m}\left(U\left(\widetilde{\mathbb{C}}_{q}^{(1)}(m)\right)\right), \forall p \in \mathbb{Z} \backslash\{0\}, j \in I .
$$

The irreducibility of the module $S\left(\hat{H}_{I}^{-}\right)$follows. The character formula (3.31) is clear. 
Remark 3.7. $R_{1}$ in Theorem 3.4 is $R_{0}$ in Theorem 2.2. If $q_{1,0}^{k} \neq 1$ for all $k \in \mathbb{N}$, then the conditions in Theorem 3.6 are satisfied. If the conditions in Theorem 3.6 are not satisfied, we do not know when the $\widetilde{\mathbb{C}}_{q}^{(1)}(m)$-module $S\left(\hat{H}_{I}^{-}\right)$is reducible except for some special cases. For example, if $n=1$, and $q_{1,0}$ is a primitive root of unity of order $m \geq 2$, we know that $S\left(\hat{H}_{I}^{-}\right)$is a highest weight module of level $m$ over the affine algebra $\widehat{g l}_{m}$ with character formula (3.31) which is the $m$-power of the character of level one highest weight module over $\widehat{g l}_{m}$. Clearly $S\left(\hat{H}_{I}^{-}\right)$is a reducible module over $\widehat{g l}_{m}$ (also over $\left.\widetilde{\mathbb{C}}_{q}^{(1)}(m)=\widetilde{\mathbb{C}}_{q}\right)$. 


\section{$\S 4$. Vertex operator representations of quantum tori at root of unity}

The quantum tori $\mathbb{C}_{q}$ studied in Theorems 2.2, 3.2, 3.4, 3.5 (also in [BGT, BS, G1, G2, GL]) have only $t_{0}$ which does not commute with the remaining commuting variables. Next we shall present vertex operator representations for quantum tori which have more non-commuting variables.

From now on in this section, we fix $I=\mathbb{Z} / m \mathbb{Z}=\{1,2, \ldots, m\}$ where $m \in \mathbb{N}$, and $\varepsilon$ in (3.5) so that

$$
\varepsilon\left(\epsilon_{i}-\epsilon_{i+1}, \epsilon_{j}-\epsilon_{j+1}\right)=\left\{\begin{array}{l}
-1, \text { if } j=i \text { or } i-1, \\
1 \text { otherwise, }
\end{array}\right.
$$

where $i=1,2, \ldots, m-1$. Let

$$
\eta\left(\epsilon_{i}-\epsilon_{j}\right)=\left\{\begin{array}{l}
-1, \text { if } j<i \\
1 \text { otherwise }
\end{array}\right.
$$

where $i, j \in\{1,2, \ldots, m\}$. It is easy to see that

$$
\begin{gathered}
\eta\left(\epsilon_{i}-\epsilon_{j}\right) \dot{E}_{i, j}^{k, l} \eta\left(\epsilon_{j}-\epsilon_{j^{\prime}}\right) \dot{E}_{j, j^{\prime}}^{l, l^{\prime}}=\eta\left(\epsilon_{i}-\epsilon_{j^{\prime}}\right) \dot{E}_{i, j^{\prime}}^{k, l^{\prime}}, \\
\eta\left(\epsilon_{i}-\epsilon_{j}\right) e_{\epsilon_{i}-\epsilon_{j}} \eta\left(\epsilon_{j}-\epsilon_{j^{\prime}}\right) e_{\epsilon_{j}-\epsilon_{j^{\prime}}}=\eta\left(\epsilon_{i}-\epsilon_{j^{\prime}}\right) e_{\epsilon_{i}-\epsilon_{j^{\prime}}} .
\end{gathered}
$$

Also from now on we fix $q=\left(q_{i, j}\right)_{i, j=0}^{n}$ satisfying $n>1$,

$$
\left\{\begin{array}{l}
q_{i, j}=q_{j, i}^{-1}, q_{i, i}=1, \forall i, j \in\{0,1, \ldots, n\}, \\
q_{i, j}=1, \forall i, j \in\{2, \ldots, n\}, \\
q_{1, i}=1, \forall i \in\{3, \ldots, n\}, \\
\text { and } q_{1,2}^{-1}=q_{2,1}=\omega_{m} \text { is a primitive root of unity of order } m .
\end{array}\right.
$$

For this $q$, generally, (1.6) and (1.6') do not define Lie algebras. We have to modify them to give Lie algebras. For this $q$ and any nonnegative integer $r$ we define $\hat{\mathbb{C}}_{q}^{(l)}(r)$ and $\tilde{\mathbb{C}}_{q}^{(l)}(r)$ by modifying (1.6') into

$$
\begin{aligned}
{\left[t^{\alpha}, t^{\beta}\right]=} & t^{\alpha} t^{\beta}-t^{\beta} t^{\alpha} \\
& +\delta_{\alpha_{0},-\beta_{0}} \delta_{\bar{\alpha}_{1},-\bar{\beta}_{1}} \delta_{\bar{\alpha}_{2},-\bar{\beta}_{2}} \delta_{\alpha+\beta, \operatorname{rad} f} \delta_{-\bar{\beta}_{l}, \bar{\alpha}_{l}} \sigma(\alpha, \beta) \alpha_{0} c, \quad \forall \alpha, \beta \in \mathbb{Z}^{n+1},
\end{aligned}
$$

where $\bar{\beta}_{l}, \bar{\alpha}_{l} \in \mathbb{Z}_{r}, \bar{\beta}_{1}, \bar{\alpha}_{1}, \bar{\beta}_{2}, \bar{\alpha}_{2} \in \mathbb{Z}_{m}$.

Theorem 4.1 Suppose $q=\left(q_{i, j}\right)_{i, j=0}^{n}$ satisfies (4.4). Set $I=\mathbb{Z} / m \mathbb{Z}=\{1,2, \ldots, m\}$. Then $\widehat{\mathbb{C}}_{q}=\widehat{\mathbb{C}}_{q}^{(1)}(0)$ has a vertex operator representation $R$ on the Fock space $V_{Q_{I}}$ so that for any $\alpha=\left(\alpha_{1}, \ldots, \alpha_{n}\right) \in \mathbb{Z}^{n}$, we have

$R\left(\sum_{p \in \mathbb{Z}}\left(t_{0}^{p} t^{\alpha}\right) z^{-p}\right)=\sum_{j=1}^{m} \eta\left(\epsilon_{j-\alpha_{1}}-\epsilon_{j}\right) \omega_{m}^{-j \alpha_{2}}\left[e_{\epsilon_{j-\alpha_{1}}-\epsilon_{j}} z^{\epsilon_{j-\alpha_{1}}-\epsilon_{j}+1-\delta_{\bar{\alpha}_{1}, 0}}\left(q^{\alpha}\right)^{-\epsilon_{j}}\left(1-q^{\alpha}\right)^{-\delta_{\bar{\alpha}_{1}, 0}}\right.$. 


$$
\begin{gathered}
\left.\cdot \exp \left(-\sum_{p \in-\mathbb{N}} \frac{\left(\epsilon_{j-\alpha_{1}}(p)-q^{-p \alpha} \epsilon_{j}(p)\right)}{p} z^{-p}\right) \exp \left(-\sum_{p \in \mathbb{N}} \frac{\left(\epsilon_{j-\alpha_{1}}(p)-q^{-p \alpha} \epsilon_{j}(p)\right)}{p} z^{-p}\right)\right] \\
\text { if } q^{\alpha} \neq 1 \text { or } \bar{\alpha}_{1} \neq 0 \\
R(c)=m, \quad R\left(t_{0}^{l} t^{\alpha}\right)=\sum_{j=1}^{m} \omega_{m}^{-j \alpha_{2}} \epsilon_{j}(l), \text { if } q^{\alpha}=1 \text { and } \bar{\alpha}_{1}=0
\end{gathered}
$$

where $\bar{\alpha}_{1} \in \mathbb{Z}_{m}$. Via (3.19), $V_{Q_{I}}$ is a weight module over $\widetilde{\mathbb{C}}_{q}$ and

$$
\operatorname{ch} V_{Q_{I}}=\left(\sum_{\alpha \in Q_{I}} x^{-(\alpha \mid \alpha) / 2}\right) \phi\left(x^{-1}\right)^{-m}
$$

Proof. It is straightforward to verify that the following linear map is a Lie algebra homomorphism:

$$
\begin{gathered}
\tau: \widehat{\mathbb{C}}_{q} \rightarrow \mathcal{A}_{I \times \infty}(\varepsilon) \\
t_{0}^{i} t^{\alpha} \rightarrow \sum_{j=1}^{m} \omega_{m}^{-j \alpha_{2}} \eta\left(\epsilon_{j-\alpha_{1}}-\epsilon_{j}\right) \sum_{k \in \mathbb{Z}} q^{-k \alpha} \dot{E}_{j-\alpha_{1}, j}^{k-i, k}+\frac{\delta_{i, 0} \delta_{\overline{\alpha_{1}}, 0} \delta_{\overline{\alpha_{2}}, 0}\left(1-\delta_{q^{\alpha}, 1}\right) m c}{1-q^{\alpha}} \\
c \rightarrow m c .
\end{gathered}
$$

If $q^{\alpha} \neq 1$, using $R_{0}$ in Theorem 3.1 we deduce that

$$
\begin{gathered}
R\left(\sum_{k \in \mathbb{Z}}\left(t_{0}^{k} t^{\alpha}\right) z^{-k}\right):=R_{0}\left(\sum_{k \in \mathbb{Z}} \tau\left(t_{0}^{k} t^{\alpha}\right) z^{-k}\right) \\
=\sum_{k \in \mathbb{Z}} z^{-k} R_{0}\left(\sum_{j=1}^{m} \omega_{m}^{-j \alpha_{2}} \eta\left(\epsilon_{j-\alpha_{1}}-\epsilon_{j}\right) \sum_{p \in \mathbb{Z}} q^{-p \alpha} \dot{E}_{j-\alpha_{1}, j}^{p-k, p}+\frac{\delta_{k, 0} \delta_{\overline{\alpha_{1}}, 0} \delta_{\overline{\alpha_{2}}, 0} m c}{1-q^{\alpha}}\right) \\
=\sum_{k \in \mathbb{Z}} z^{-k} \sum_{j=1}^{m} \omega_{m}^{-j \alpha_{2}} \eta\left(\epsilon_{j-\alpha_{1}}-\epsilon_{j}\right)\left(R_{0}\left(\sum_{p \in \mathbb{Z}}\left(q^{-p \alpha} \dot{E}_{j-\alpha_{1}, j}^{p-k, p}\right)+\frac{\delta_{k, 0} \delta_{\overline{\alpha_{1}}, 0} c}{1-q^{\alpha}}\right)\right) \\
=\sum_{j=1}^{m} \omega_{m}^{-j \alpha_{2}} \eta\left(\epsilon_{j-\alpha_{1}}-\epsilon_{j}\right)\left[e_{\epsilon_{j-\alpha_{1}}-\epsilon_{j}} z^{\epsilon_{j-\alpha_{1}}-\epsilon_{j}+1-\delta_{\bar{\alpha}_{1}, 0}\left(q^{\alpha}\right)^{-\epsilon_{j}}\left(1-q^{\alpha}\right)^{-\delta_{\bar{\alpha}_{1}, 0}}}\right. \\
\left.\cdot \exp \left(-\sum_{p \in-\mathbb{N}} \frac{\left(\epsilon_{j-\alpha_{1}}(p)-q^{-p \alpha} \epsilon_{j}(p)\right)}{p} z^{-p}\right) \exp \left(-\sum_{p \in \mathbb{N}} \frac{\left(\epsilon_{j-\alpha_{1}}(p)-q^{-p \alpha} \epsilon_{j}(p)\right)}{p} z^{-p}\right)\right] .
\end{gathered}
$$


The other formulas of (4.6) are quite clear. To see that $V_{Q_{I}}$ is a weight module over $\widetilde{\mathbb{C}_{q}}$, we need to show that

$$
\left[R\left(d_{0}\right), R\left(\sum_{p \in \mathbb{Z}}\left(t_{0}^{p} t^{\alpha}\right) z^{-p}\right)\right]=R\left(\sum_{p \in \mathbb{Z}}\left(t_{0}^{p} t^{\alpha}\right) p z^{-p}\right)=-\frac{z \partial}{\partial z} R\left(\sum_{p \in \mathbb{Z}}\left(t_{0}^{p} t^{\alpha}\right) z^{-p}\right)
$$

similar to Theorem 3.4, which can be verified by using (3.17) and

$$
z \frac{\partial}{\partial z} z^{\alpha}=\alpha(0) z^{\alpha}, \quad \forall \alpha \in Q_{I}
$$

Other parts of the theorem follows easily. Thus we complete the proof of this theorem.

Remark 4.2. The embedding (4.8) without center is inspired by the following string

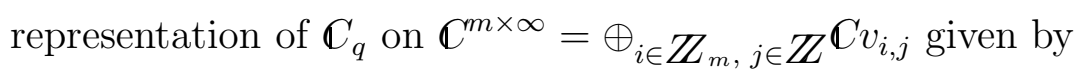

$$
\left(t_{0}^{p} t^{\alpha}\right) v_{i, j}=\omega_{m}^{-i \alpha_{2}} q^{-j \alpha} v_{i-\alpha_{1}, j-p}
$$

Remark 4.3. The Lie algebras considered in Theorems 3.2 and 4.1 are not isomorphic. Indeed, it follows by examining the maximal abelian subalgebras $M$ satisfying $c \notin\left[\hat{\mathbb{C}}_{q}, M\right]$. But some of them have isomorphic quotients.

Theorem 4.4 In addition to the assumptions in Theorem 4.1, if one of the following holds then $\widetilde{\mathbb{C}}_{q}$-module $V_{Q_{I}}$ in Theorem 4.1 is irreducible:

(1) $\left\{q^{\alpha}=q_{1,0}^{\alpha_{1}} \ldots q_{n, 0}^{\alpha_{n}} \mid \alpha=\left(\alpha_{1}, \alpha_{2}, \ldots, \alpha_{n}\right) \in \mathbb{Z}^{n}\right\}$ is infinite;

(2) there exists $\alpha=\left(\alpha_{1}, \ldots, \alpha_{n}\right) \in \mathbb{Z}^{n}$ such that $m \mid \alpha_{1},\left(\alpha_{2}, m\right)=1$ and $q^{\alpha}=1$.

Proof. Claim 1. $\epsilon_{j}(l) \in U\left(R_{m}\left(\widetilde{\mathbb{C}_{q}}\right)\right)$, the universal enveloping algebra of $R_{m}\left(\widetilde{\mathbb{C}_{q}}\right), \quad \forall j \in$ $I, l \in \mathbb{Z} \backslash\{0\}$.

If Condition (2) holds, Claim 1 follows from the third equation of (4.6), furthermore, $\epsilon_{j}(0) \in U\left(R_{m}\left(\widetilde{\mathbb{C}_{q}}\right)\right), \quad \forall j \in I$. Next we suppose Condition (1) holds. Fix $l \in \mathbb{Z}$. Then the set $\left\{q^{\alpha}=q_{1,0}^{\alpha_{1}} \ldots q_{n, 0}^{\alpha_{n}} \mid \alpha=\left(\alpha_{1}, \ldots, \alpha_{n}\right) \in \mathbb{Z}^{n}\right.$ with $\left.m \mid \alpha_{1}-l\right\}$ is infinite. For each $\alpha=\left(\alpha_{1}, \ldots \alpha_{n}\right) \in$ $\mathbb{Z}^{n}$ with $m \mid \alpha_{1}-l$, let

$$
\begin{gathered}
f_{j}\left(q^{\alpha}, l, z\right)=\eta\left(\epsilon_{j-\alpha_{1}}-\epsilon_{j}\right) \epsilon_{\epsilon_{j-\alpha_{1}}-\epsilon_{j}} z^{\epsilon_{j-\alpha_{1}}-\epsilon_{j}+1-\delta_{\bar{\alpha}_{1}, 0}}\left(q^{\alpha}\right)^{-\epsilon_{j}}\left(1-q^{\alpha}\right)^{-\delta_{\bar{\alpha}_{1}, 0}} . \\
\cdot \exp \left(-\sum_{p \in-\mathbb{N}} \frac{\left(\epsilon_{j-\alpha_{1}}(p)-q^{-p \alpha} \epsilon_{j}(p)\right)}{p} z^{-p}\right) \exp \left(-\sum_{p \in \mathbb{N}} \frac{\left(\epsilon_{j-\alpha_{1}}(p)-q^{-p \alpha} \epsilon_{j}(p)\right)}{p} z^{-p}\right),
\end{gathered}
$$


where $q^{\alpha}=q_{1,0}^{\alpha_{1}} \ldots q_{n, 0}^{\alpha_{n}}$ and we require $q^{\alpha} \neq 1$ if $\bar{\alpha}_{1}=0$. For any $v \in V_{Q_{I}}$, from (4.6) we know that

$$
\sum_{j=1}^{m} \omega_{m}^{-j \alpha_{2}} f_{j}\left(q^{\alpha}, l, z\right) v \in\left(R_{m}\left(\widetilde{\mathbb{C}_{q}}\right) v\right)\left[z, z^{-1}\right], \quad \forall l \in \mathbb{Z}, \alpha \in \mathbb{Z}^{n}
$$

Note that $f_{j}\left(q^{\alpha}, l, z\right) v \in V_{Q_{I}}\left[z, z^{-1}\right]$ is indeed a vector valued function of $q^{\alpha}$. Thus using Lemma 3.5, we see that, for any $j$,

$$
f_{j}\left(q^{\alpha}, l, z\right) v \in\left(R_{m}\left(\widetilde{\mathbb{C}_{q}}\right) v\right)\left[z, z^{-1}\right], \quad \forall l \in \mathbb{Z}, \alpha \in \mathbb{Z}^{n}, v \in V_{Q_{I}} .
$$

It is clear that, for any $j$,

$$
\begin{aligned}
& {\left[f_{j}\left(q^{\alpha}, 0, z_{1}\right), f_{j}\left(q^{-\alpha}, 0, z_{2}\right)\right] v \in\left(R_{m}\left(U\left(\widetilde{\mathbb{C}_{q}}\right)\right) v\right)\left[z_{1}^{ \pm 1}, z_{2}^{ \pm 1}\right]} \\
& \forall \alpha \in \mathbb{Z}^{n} \quad \text { with } m \mid \alpha_{1}, q_{1,0}^{\alpha_{1}} \ldots q_{n, 0}^{\alpha_{n}} \neq 1, \quad \forall v \in V_{Q_{I}}
\end{aligned}
$$

As in the proof of Theorem 3.6, we have

$$
\left[f_{j}\left(q^{\alpha}, 0, z_{1}\right), f_{j}\left(q^{-\alpha}, 0, z_{2}\right)\right]=\sum_{p, p^{\prime} \in \mathbb{Z}} q^{-p \alpha}\left(q^{\left(p+p^{\prime}\right) \alpha}-1\right) \epsilon_{j}\left(p+p^{\prime}\right) z_{1}^{-p} z_{2}^{-p^{\prime}}+(D \delta)\left(\frac{z_{2}}{q^{\alpha} z_{1}}\right) .
$$

Since Condition (1) holds, then

$$
\epsilon_{j}(p) \in R_{m}(U(L)), \quad \forall p \in \mathbb{Z} \backslash\{0\}, j \in I
$$

Claim 1 follows as well.

Claim 2. $e_{\epsilon_{i}-\epsilon_{j}} z^{\epsilon_{i}-\epsilon_{j}} q_{1,0}^{(i-j) \epsilon_{j}} \in U\left(R_{m}\left(\widetilde{\mathbb{C}_{q}}\right)\right)\left[z, z^{-1}\right], \quad \forall i, j \in I$.

Equation (4.10) with $\bar{\alpha}_{1} \neq 0$ holds even when Condition (2) is true. Indeed, it follows from the fact

$$
\operatorname{ad}\left(\epsilon_{j}(0)\right)^{k} R\left(\sum_{p \in \mathbb{Z}}\left(t_{0}^{p} t^{\alpha}\right) z^{-p}\right) \in\left(U\left(R_{m}\left(\widetilde{\mathbb{C}_{q}}\right)\right) v\right)\left[z, z^{-1}\right], \quad \forall k \in \mathbb{N}, \alpha \in \mathbb{Z}^{n} \text { with } \bar{\alpha}_{1} \neq 0
$$

From (4.10) with $\bar{\alpha}_{1} \neq 0$ and Claim 1 , we know that, if $\bar{\alpha}_{1} \neq 0$, for any $v \in V_{Q_{I}}$,

$$
\begin{aligned}
& e_{\epsilon_{j-\alpha_{1}}-\epsilon_{j}} z^{\epsilon_{j-\alpha_{1}}-\epsilon_{j}} q_{1,0}^{-\alpha_{1} \epsilon_{j}} v=\eta\left(\epsilon_{j-\alpha_{1}}-\epsilon_{j}\right) z^{-1} \exp \left(\sum_{p \in-\mathbb{N}} \frac{\left(\epsilon_{j-\alpha_{1}}(p)-q^{-p\left(\alpha_{1}, 0 \ldots 0\right)} \epsilon_{j}(p)\right)}{p} z^{-p}\right) . \\
& \cdot f_{j}\left(q^{\left(\alpha_{1}, 0 \ldots 0\right)}, \alpha_{1}, z\right) \exp \left(\sum_{p \in \mathbb{N}} \frac{\left(\epsilon_{j-\alpha_{1}}(p)-q^{-p\left(\alpha_{1}, 0 \ldots 0\right)} \epsilon_{j}(p)\right)}{p} z^{-p}\right) v \in\left(U\left(R_{m}\left(\widetilde{\mathbb{C}_{q}}\right)\right) v\right)\left[z, z^{-1}\right] .
\end{aligned}
$$


Thus

$$
e_{\epsilon_{j-\alpha_{1}}-\epsilon_{j}} z^{\epsilon_{j-\alpha_{1}}-\epsilon_{j}} q_{1,0}^{-\alpha_{1} \epsilon_{j}} \in\left(U\left(R_{m}\left(\widetilde{\mathbb{C}_{q}}\right)\right)\right)\left[z, z^{-1}\right]
$$

Claim 2 follows.

Now we are ready to show the irreducibility of $\widetilde{\mathbb{C}_{q}}$-module $V_{Q_{I}}$. Suppose $W$ is a nonzero $\widetilde{\mathbb{C}_{q}}$-submodule of $V_{Q_{I}}$. Then $d_{0}$ acts diagonally on $W$. From Claim 1 we can choose a nonzero weight element in $W$ of the form

$$
w=\sum_{i=1}^{r} u_{i} \otimes e^{\gamma_{i}} \in W
$$

where $u_{i} \in S\left(\hat{H}_{I}^{-}\right), \gamma_{i} \in Q_{I}$, such that $r$ is minimal.

Suppose $r \geq 2$. We see that any two of $\gamma_{i}$ 's are distinct and

$$
\operatorname{deg}\left(u_{1}\right)-\frac{\left(\gamma_{1} \mid \gamma_{1}\right)}{2}=\operatorname{deg}\left(u_{i}\right)-\frac{\left(\gamma_{i} \mid \gamma_{i}\right)}{2}, \forall i=1, \ldots, r
$$

Since $\gamma_{1} \neq \gamma_{2}$, there exist $k, l \in I$ such that

$$
\left(\epsilon_{k}-\epsilon_{l} \mid \gamma_{1}\right) \neq\left(\epsilon_{k}-\epsilon_{l} \mid \gamma_{2}\right)
$$

From Claim 2, we deduce that

$$
e_{\epsilon_{k}-\epsilon_{l}} z^{\epsilon_{k}-\epsilon_{l}} q_{1,0}^{(l-k) \epsilon_{j}} w=\sum_{i=1}^{r} \varepsilon\left(\epsilon_{k}-\epsilon_{l}, \gamma_{i}\right) z^{\left(\epsilon_{k}-\epsilon_{l} \mid \gamma_{i}\right)} q_{1,0}^{(l-k)\left(\epsilon_{j} \mid \gamma_{i}\right)} u_{i} \otimes e^{\epsilon_{k}-\epsilon_{l}+\gamma_{i}} \in W\left[z, z^{-1}\right]
$$

Since the powers of $z$ in the right hand side of (4.13) are not the same, we get a nonzero element in $W$ which has expression like in (4.13) with smaller $r$. This contradicts the minimality of $r$. Consequently $r=1$. From Claims 1 and 2, we know that any nonzero element $u \otimes e^{\gamma}$ can generate the whole $\widetilde{\mathbb{C}}_{q}$-module $V_{Q_{I}}$, where $u \in S\left(\hat{H}_{I}^{-}\right), \gamma \in Q$, thus $W=V_{Q_{I}}$. This completes the proof of this theorem.

Next we shall construct level $m r$ vertex operator representations for the algebra $\widehat{\mathbb{C}}_{q}^{(l)}(r)$ for any positive integer $r$ defined in (4.5). For any $k \in\{1,2, \ldots, r\}$, let $I=I_{k}=\{1,2, \ldots, m\}$. For each $I_{k}$, along from (3.3) through (3.21), similarly we define $P_{I_{k}}=\oplus_{j \in I} \mathbb{Z} \epsilon_{j}^{(k)}$ with the standard bilinear form, $Q_{I^{(k)}}=\oplus_{i, j \in I_{i}} \mathbb{Z}\left(\epsilon_{i}^{(k)}-\epsilon_{j}^{(k)}\right), H_{I^{(k)}}$ and $\hat{H}_{I^{(k)}}$ (with the same $c, d_{0}$ ), the operators $e_{\beta} \in \operatorname{End} \mathbb{C}\left[Q_{I^{(k)}}\right]$ for any $\beta \in Q_{I^{(k)}}$, the operators $\beta(0) \in \operatorname{End} \mathbb{C}\left[Q_{I^{(k)}}\right]$ for any $\beta \in H_{I^{(k)}}$, the operators $z^{\beta} \in \operatorname{End} \mathbb{C}\left[Q_{I^{(k)}}\right][[z]]$ for any $\beta \in Q_{I^{(k)}}$ (operators for different $k$ are commutative), and the vector space

$$
V_{Q_{I^{(k)}}}=\mathbb{C}\left[Q_{I^{(k)}}\right] \otimes S\left(H_{I^{(k)}}^{-}\right)
$$


Then we have the tensor product

$$
\left(V_{Q_{I}}\right)^{r}=\otimes_{i=1}^{r} V_{Q_{I^{(k)}}}
$$

Thus we have the representation $R^{(k)}$ of ${\widehat{\mathbb{C}_{q}}}^{(l)}(0)$ on each $V_{Q_{I^{(k)}}}$ in the sense of Theorem 4.1.

Theorem 4.12 Suppose $\omega_{m}, q=\left(q_{i, j}\right)_{i, j=0}^{n}$ are given as in Theorem $3.8, l \in\{1,2, \ldots, n\}$. For any positive integer $r$, let $I_{i}=\mathbb{Z} / m \mathbb{Z}=\{1,2, \ldots, m\}$ for $i \in\{1,2, \ldots, r\}$, let $\omega_{r}$ be the primitive root of unity of order $r$. Then $\widehat{\mathbb{C}}_{q}^{(l)}(r)$ has a vertex operator representation $R_{r}^{(l)}$ on the Fock space (4.15) so that for any $\alpha=\left(\alpha_{1}, \ldots, \alpha_{n}\right) \in \mathbb{Z}^{n}$, we have

$$
\begin{aligned}
& R_{r}^{(l)}\left(\sum_{p \in \mathbb{Z}}\left(t_{0}^{p} t^{\alpha}\right) z^{-p}\right)=\sum_{k=1}^{r} \omega_{r}^{-k \alpha_{l}} \sum_{j=1}^{m} \eta\left(\epsilon_{j-\alpha_{1}}^{(k)}-\epsilon_{j}^{(k)}\right) \omega_{m}^{-j \alpha_{2}} \\
& \cdot\left[e_{\epsilon_{j-\alpha_{1}}^{(k)}-\epsilon_{j}^{(k)}} z^{\epsilon_{j-\alpha_{1}}^{(k)}-\epsilon_{j}^{(k)}+1-\delta_{\bar{\alpha}_{1}, 0}}\left(q^{\alpha}\right)^{-\epsilon_{j}^{(k)}}\left(1-q^{\alpha}\right)^{-\delta_{\bar{\alpha}_{1}, 0}} \cdot\right. \\
& \left.\cdot \exp \left(-\sum_{p \in-\mathbb{N}} \frac{\left(\epsilon_{j-\alpha_{1}}^{(k)}(p)-q^{-p \alpha} \epsilon_{j}^{(k)}(p)\right)}{p} z^{-p}\right) \exp \left(-\sum_{p \in \mathbb{N}} \frac{\left(\epsilon_{j-\alpha_{1}}^{(k)}(p)-q^{-p \alpha} \epsilon_{j}^{(k)}(p)\right)}{p} z^{-p}\right)\right], \\
& \text { if } q^{\alpha} \neq 1 \text { or } \bar{\alpha}_{1} \neq 0 \text {, } \\
& R_{r}^{(l)}(c)=m r, \quad R_{r}^{(l)}\left(t_{0}^{i} t^{\alpha}\right)=\sum_{k=1}^{r} \omega_{r}^{-k \alpha_{l}} \sum_{j=1}^{m} \omega_{m}^{-j \alpha_{2}} \epsilon_{j}^{(k)}(i), \text { if } q^{\alpha}=1 \text { and } \bar{\alpha}_{1}=0
\end{aligned}
$$

where $\bar{\alpha}_{1} \in \mathbb{Z}_{m}$. Via (3.19), $\left(V_{Q_{I}}\right)^{r}$ is a weight module over $\widetilde{\mathbb{C}}_{q}^{(l)}(r)$ and

$$
\operatorname{ch}\left(V_{Q_{I}}\right)^{r}=\left(\sum_{\alpha \in Q_{I}} x^{-(\alpha \mid \alpha) / 2}\right)^{r} \phi\left(x^{-1}\right)^{-m r} .
$$

Proof. We denote by $R^{(k)}$ the vertex operator representation of $\widehat{\mathbb{C}}_{q}=\widehat{\mathbb{C}}_{q}^{(1)}(0)$ on $V_{Q_{I^{(k)}}}$ in the sense of Theorem 4.1. We still use $R^{(k)}$ to denote the extended representation of $\widehat{\mathbb{C}_{q}}$ on $\otimes_{i=1}^{r} V_{Q_{I}(k)}$, for example,

$$
\begin{gathered}
R^{(1)}\left(t^{\alpha}\right)=R^{(1)}\left(t^{\alpha}\right) \otimes 1 \otimes \ldots \otimes 1, \\
R^{(2)}\left(t^{\alpha}\right)=1 \otimes R^{(1)}\left(t^{\alpha}\right) \otimes 1 \otimes \ldots \otimes 1 .
\end{gathered}
$$

Thus we have

$$
\left[R^{(k)}\left(t^{\alpha}\right), R^{(j)}\left(t^{\alpha^{\prime}}\right)\right]=\delta_{k, j}\left[\left(\sigma\left(\alpha, \alpha^{\prime}\right)-\sigma\left(\alpha^{\prime}, \alpha\right)\right) R^{(k)}\left(t^{\alpha+a^{\prime}}\right)\right.
$$




$$
\left.+\delta_{\alpha+\alpha^{\prime}, \operatorname{rad} f} \delta_{\alpha_{0},-\alpha_{0}^{\prime}} \delta_{\bar{\alpha}_{1},-\bar{\alpha}_{1}^{\prime}} \delta_{\bar{\alpha}_{2},-\bar{\alpha}_{2}^{\prime}} \sigma\left(\alpha, \alpha^{\prime}\right) m \alpha_{0}\right], \quad \forall \alpha, \alpha^{\prime} \in \mathbb{Z}^{n+1}
$$

From (4.6) we know that

$$
R_{r}\left(t^{\alpha}\right)=\sum_{k=1}^{r} \omega_{r}^{-k \alpha_{l}} R^{(k)}\left(t^{\alpha}\right) \forall \alpha \in \mathbb{Z}^{n+1}
$$

Then for any $\alpha, \alpha^{\prime} \in \mathbb{Z}^{n+1}$ we deduce that

$$
\begin{gathered}
{\left[R_{r}\left(t^{\alpha}\right), R_{r}\left(t^{\alpha^{\prime}}\right)\right]=\left[\sum_{k=1}^{r} \omega_{r}^{-k \alpha_{l}} R^{(k)}\left(t^{\alpha}\right), \sum_{k=1}^{r} \omega_{r}^{-k \alpha_{l}^{\prime}} R^{(k)}\left(t^{\alpha^{\prime}}\right)\right]} \\
=\sum_{k=1}^{r} \omega_{r}^{-k\left(\alpha_{l}+\alpha_{l}^{\prime}\right)}\left[R^{(k)}\left(t^{\alpha}\right), R^{(k)}\left(t^{\alpha^{\prime}}\right)\right] \\
=\sum_{k=1}^{r} \omega_{r}^{-k\left(\alpha_{l}+\alpha_{l}^{\prime}\right)}\left[\left(\sigma\left(\alpha, \alpha^{\prime}\right)-\sigma\left(\alpha^{\prime}, \alpha\right)\right) R^{(k)}\left(t^{\alpha+a^{\prime}}\right)+\delta_{\alpha+\alpha^{\prime}, \operatorname{rad} f} \delta_{\alpha_{0},-\alpha_{0}^{\prime}} \delta_{\bar{\alpha}_{1},-\bar{\alpha}_{1}^{\prime}} \delta_{\bar{\alpha}_{2},-\bar{\alpha}_{2}^{\prime}} \sigma\left(\alpha, \alpha^{\prime}\right) m \alpha_{0}\right] \\
=\left(\sigma\left(\alpha, \alpha^{\prime}\right)-\sigma\left(\alpha^{\prime}, \alpha\right)\right) R_{r}^{(l)}\left(t^{\alpha+a^{\prime}}\right)+\delta_{\alpha+\alpha^{\prime}, \operatorname{rad} f} \delta_{\bar{\alpha}_{l},-\bar{\alpha}_{l}^{\prime}} \delta_{\alpha_{0},-\alpha_{0}^{\prime}} \delta_{\bar{\alpha}_{1},-\bar{\alpha}_{1}^{\prime}} \delta_{\bar{\alpha}_{2},-\bar{\alpha}_{2}^{\prime}} \sigma\left(\alpha, \alpha^{\prime}\right) m r \alpha_{0} \\
=R_{r}^{(l)}\left(\left(\sigma\left(\alpha, \alpha^{\prime}\right)-\sigma\left(\alpha^{\prime}, \alpha\right)\right) t^{\alpha+a^{\prime}}+\delta_{\alpha+\alpha^{\prime}, \operatorname{rad} f} \delta_{\bar{\alpha}_{l},-\bar{\alpha}_{l}^{\prime}} \delta_{\alpha_{0},-\alpha_{0}^{\prime}} \delta_{\bar{\alpha}_{1},-\bar{\alpha}_{1}^{\prime}} \delta_{\bar{\alpha}_{2},-\bar{\alpha}_{2}^{\prime}} \sigma\left(\alpha, \alpha^{\prime}\right) \alpha_{0} c\right) \\
=R_{r}^{(l)}\left(\left[t^{\alpha}, t^{\alpha^{\prime}}\right]\right),
\end{gathered}
$$

where $\bar{\alpha}_{l},-\bar{\alpha}_{l}^{\prime} \in \mathbb{Z}_{r} \bar{\alpha}_{1}, \bar{\alpha}_{1}^{\prime}, \bar{\alpha}_{2},-\bar{\alpha}_{2}^{\prime} \in \mathbb{Z}_{m}$ and the last bracket is in $\widetilde{\mathbb{C}}_{q}^{(l)}(r)$. So $R_{r}^{(l)}$ is a representation of ${\widetilde{\mathbb{C}_{q}}}^{(l)}(r)$. The character formula (4.17) is clear. Thus we proved the theorem.

Like in Remark 3.7 for $R_{m}$ in Theorem 3.4, some representations $R_{r}^{(l)}$ of $\widetilde{\mathbb{C}}_{q}^{(l)}(r)$ are irreducible. Now we give some sufficient conditions for $R_{r}^{(l)}$ to be irreducible.

Theorem 4.13 The vertex operator representation $R_{r}^{(l)}$ of $\widetilde{\mathbb{C}}_{q}^{(l)}(r)$ in Theorem 4.12 is irreducible if one of the following holds:

(1) $(m, r)=1$ and there exists $\alpha=\left(\alpha_{1}, \ldots, \alpha_{n}\right) \in \mathbb{Z}^{n}$ such that $m \mid \alpha_{1},\left(\alpha_{l}, r\right)=\left(\alpha_{2}, m\right)=1$, and $q^{\alpha}=1$;

(2) $l>2$ and the set $\left\{q^{\alpha}=q_{1,0}^{\alpha_{1}} \ldots q_{n, 0}^{\alpha_{n}} \mid \alpha=\left(\alpha_{1}, \ldots, \alpha_{n}\right) \in \mathbb{Z}^{n}\right\}$ is infinite;

(3) $l \in\{1,2\},(m, r)=1$ and the set $\left\{q^{\alpha}=q_{1,0}^{\alpha_{1}} \ldots q_{n, 0}^{\alpha_{n}} \mid \alpha=\left(\alpha_{1}, \ldots, \alpha_{n}\right) \in \mathbb{Z}^{n}\right\}$ is infinite.

Proof. The proof of this theorem is very similar to that of Theorem 3.6. So here we only outline the proof for Cases (1) and (2). 
(1) From the hypothesis and the third equation of (4.16), we deduce that $\epsilon_{j}^{(k)}(i) \in$ $U\left(R_{r}^{(l)}\left(\widetilde{\mathbb{C}}_{q}^{(l)}(r)\right)\right)$, the universal enveloping algebra of $R_{r}^{(l)}\left(\widetilde{\mathbb{C}}_{q}^{(l)}(r)\right), \quad \forall j \in I, i \in \mathbb{Z}, k \in$ $\{1,2, \ldots, r\}$. From

$$
\operatorname{ad}\left(\epsilon_{j}^{(k)}(0)\right)^{i} R_{r}^{(l)}\left(\sum_{p \in \mathbb{Z}}\left(t_{0}^{p} t^{\alpha}\right) z^{-p}\right) \in U\left(R_{r}^{(l)}\left(\widetilde{\mathbb{C}}_{q}^{(l)}(r)\right)\right)\left[z, z^{-1}\right], \quad \forall i \in \mathbb{N}, \alpha \in \mathbb{Z}^{n} \text { with } \alpha_{1} \neq 0
$$

we deduce that, if $\bar{\alpha}_{1} \neq 0$, then

$$
\begin{aligned}
& f_{j}^{(k)}(\alpha, z)=e_{\epsilon_{j-\alpha_{1}}^{(k)}-\epsilon_{j}^{(k)}} z^{\epsilon_{j-\alpha_{1}}^{(k)}-\epsilon_{j}^{(k)}+1}\left(q^{\alpha}\right)^{-\epsilon_{j}^{(k)}} . \\
& \cdot \exp \left(-\sum_{p \in-\mathbb{N}} \frac{\left(\epsilon_{j-\alpha_{1}}^{(k)}(p)-q^{-p \alpha} \epsilon_{j}^{(k)}(p)\right)}{p} z^{-p}\right) \exp \left(-\sum_{p \in \mathbb{N}} \frac{\left(\epsilon_{j-\alpha_{1}}^{(k)}(p)-q^{-p \alpha} \epsilon_{j}^{(k)}(p)\right)}{p} z^{-p}\right) \\
& \in U\left(R_{r}^{(l)}\left(\widetilde{\mathbb{C}}_{q}^{(l)}(r)\right)\right)\left[z, z^{-1}\right] \\
& e_{\epsilon_{j-\alpha_{1}}^{(k)}-\epsilon_{j}^{(k)}} z^{\epsilon_{j-\alpha_{1}}^{(k)}-\epsilon_{j}^{(k)}} q_{1,0}^{-\alpha_{1} \epsilon_{j}^{(k)}}=z^{-1} \exp \left(\sum_{p \in-I N} \frac{\left(\epsilon_{j-\alpha_{1}}^{(k)}(p)-q_{1,0}^{-p \alpha_{1}} \epsilon_{j}^{(k)}(p)\right)}{p} z^{-p}\right) . \\
& \cdot f_{j}^{(k)}\left(\left(\alpha_{1}, 0, \ldots, 0\right), z\right) \exp \left(\sum_{p \in \mathbb{N}} \frac{\left(\epsilon_{j-\alpha_{1}}^{(k)}(p)-q_{1,0}^{-p \alpha_{1}} \epsilon_{j}^{(k)}(p)\right)}{p} z^{-p}\right) \in U\left(R_{m}\left(\widetilde{\mathbb{C}_{q}}\right)\right)\left[z, z^{-1}\right] .
\end{aligned}
$$

Thus

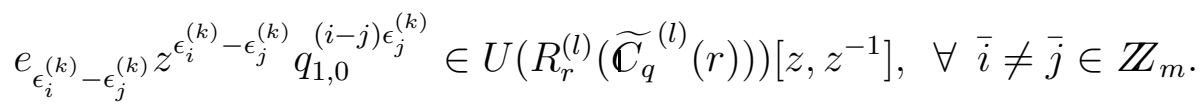

Suppose $W$ is a nonzero $\widetilde{\mathbb{C}}_{q}^{(l)}(r)$-submodule of $\left(V_{Q_{I}}\right)^{r}$. Then $d_{0}$ acts diagonally on $W$. We can choose a nonzero weight element in $W$ of the form

$$
w=\sum_{i=1}^{r} u_{i} \otimes e^{\gamma_{i}} \in W
$$

where $u_{i} \in S\left(\otimes_{j=1}^{r} \hat{H}_{I_{j}}^{-}\right), \gamma_{i} \in \otimes_{j=1}^{r} Q_{I^{(j)}}$, such that $r$ is minimal.

Repeat the last paragraph of the proof of Theorem 4.12 we see that $W=\left(V_{Q_{I}}\right)^{r}$.

(2) From the hypothesis we know that for any fixed $s_{1}, s_{2}, s_{l}$, the set $\left\{q^{\alpha}=q_{1,0}^{\alpha_{1}} \ldots q_{n, 0}^{\alpha_{n}} \mid \alpha=\right.$ $\left(\alpha_{1}, \ldots, \alpha_{n}\right) \in \mathbb{Z}^{n}$ with $\left.\alpha_{1}=s_{1}(\bmod m), \alpha_{2}=s_{2}(\bmod m), \alpha_{l}=s_{l}(\bmod r)\right\}$ is infinite. Using Lemma 3.5 we deduce that

$$
f_{j}^{(k)}(\alpha, z)=e_{\epsilon_{j-\alpha_{1}}^{(k)}-\epsilon_{j}^{(k)}} z^{\epsilon_{j-\alpha_{1}}^{(k)}-\epsilon_{j}^{(k)}+1-\delta_{\bar{\alpha}_{1}, 0}}\left(q^{\alpha}\right)^{-\epsilon_{j}^{(k)}}\left(1-q^{\alpha}\right)^{-\delta_{\bar{\alpha}_{1}, 0}}
$$




$$
\begin{gathered}
\cdot \exp \left(-\sum_{p \in-\mathbb{N}} \frac{\left(\epsilon_{j-\alpha_{1}}^{(k)}(p)-q^{-p \alpha} \epsilon_{j}^{(k)}(p)\right)}{p} z^{-p}\right) \exp \left(-\sum_{p \in \mathbb{N}} \frac{\left(\epsilon_{j-\alpha_{1}}^{(k)}(p)-q^{-p \alpha} \epsilon_{j}^{(k)}(p)\right)}{p} z^{-p}\right) \\
\in U\left(R_{r}^{(l)}\left(\widetilde{\mathbb{C}_{q}}\right)\right)\left[z, z^{-1}\right], \quad \forall \alpha \in \mathbb{Z}^{n} \text { with } q^{\alpha} \neq 1 \text { or } \bar{\alpha}_{1} \neq 0 .
\end{gathered}
$$

For any $\alpha \in \mathbb{Z}^{n}$ with $\alpha_{1}=0(\bmod m)$ and $q^{\alpha} \neq 1$, similar to $(4.12)$ by computing

$$
\left[f_{j}^{(k)}\left(\alpha, z_{1}\right), f_{j}^{(k)}\left(-\alpha, z_{2}\right)\right] \in U\left(R_{r}^{(l)}\left(\widetilde{\mathbb{C}_{q}}\right)\right)\left[z, z^{-1}\right]
$$

we deduce that

$$
\epsilon_{j}^{(k)}(p) \in R_{m}(U(L)), \quad \forall p \in \mathbb{Z} \backslash\{0\}, j, k
$$

Similar to Claim 2 in the proof of Theorem 4.11, we can also show that

$$
e_{\epsilon_{i}^{(k)}-\epsilon_{j}^{(k)}} z^{\epsilon_{i}^{(k)}-\epsilon_{j}^{(k)}} q_{1,0}^{(i-j) \epsilon_{j}^{(k)}} \in U\left(R_{r}^{(l)}\left(\widetilde{\mathbb{C}_{q}}\right)\right)\left[z, z^{-1}\right], \quad \forall i, j \in I, \quad \forall k
$$

Then we repeat the remaining discussion for Condition (1) to see the irreducibility of the representation.

Remark 4.14 If we assume that $q_{1,0}^{\alpha_{1}} \ldots q_{n, 0}^{\alpha_{n}}=1$ implies $\alpha=\left(\alpha_{1}, \alpha_{2}, \ldots, \alpha_{n}\right)=0$, then $\widetilde{\mathbb{C}}_{q}^{(l)}(r) \simeq \tilde{\mathbb{C}}_{q}^{(l)}(r) \simeq \tilde{\mathbb{C}}_{q}$ and $\widehat{\mathbb{C}}_{q}^{(l)}(r) \simeq \hat{\mathbb{C}}_{q}^{(l)}(r) \simeq \hat{\mathbb{C}}_{q}$ for all the algebras discussed in this section, and all the modules constructed in this paper for these algebras are irreducible.

\section{§5. Highest weight modules}

For brevity, we denote $L=\hat{\mathbb{C}}_{q}, \tilde{\mathbb{C}}_{q}, \hat{\mathbb{C}}_{q}^{(l)}(r), \tilde{\mathbb{C}}_{q}^{(l)}(r)$, defined by (1.6) and (1.6') under the condition (1.5), or defined by (4.5) under the condition (4.4). With respect to $d_{0}$, $L$ has the $\mathbb{Z}$-gradation (1.7). Let $L_{+}=\oplus_{i \in \mathbb{Z}_{+}} L_{i}, L_{-}=\oplus_{i<0} L_{i}$.

Now we define highest weight modules over $L$. Suppose $\dot{c}, \dot{d} \in \mathbb{C}, \lambda=\left(\lambda_{\alpha}\right)$ where $\lambda_{\alpha} \in \mathbb{C}$, $\alpha=\left(\alpha_{1}, \ldots, \alpha_{n}\right) \in \mathbb{Z}^{n}\left(\lambda_{\alpha} \in \mathbb{C}\right.$ can be arbitrary if $\left.q^{\alpha}=1\right)$. We can define the 1 -dimensional $L_{+}$-module $\mathbb{C} v_{0}$ via

$$
L_{i} v_{0}=0, \text { if } i>0 ; c v_{0}=\dot{c} v_{0}, d v=\dot{d} v_{0}, t^{\alpha} v_{0}=\lambda_{\alpha} v_{0}, \quad \forall \alpha \in \mathbb{Z}^{n}
$$

This implies that $\left[L_{0}, L_{0}\right] v_{0}=0$. Then we have the induced $L$-module

$$
\bar{V}(\lambda, \dot{c}, \dot{d})=\operatorname{Ind}_{L}^{L_{+}} \mathbb{C} v_{0}=U(L) \otimes_{U\left(L_{+}+L_{0}\right)} \mathbb{C} v_{0},
$$

where $U(L)$ is the universal enveloping algebra of the Lie algebra $L$. It is clear that, as vector spaces, $\bar{V}(\lambda, \dot{c}, \dot{d}) \simeq U\left(L_{-}\right)$. The module $\bar{V}(\lambda, \dot{c}, \dot{d})$ has a unique maximal proper submodule 
$J$. Then we obtain the irreducible module

$$
V(\lambda, \dot{c}, \dot{d})=\frac{\bar{V}(\lambda, \dot{c}, \dot{d})}{J}
$$

It is clear that $V(\lambda, \dot{c}, \dot{d})$ is uniquely determined by the parameters $\lambda, \dot{c}, \dot{d}$. Since the structure of $V(\lambda, \dot{c}, \dot{d})$ is independent of $\dot{d}$, so we shall always assume that $\dot{d}=0$, and simply denote $V(\lambda, \dot{c}, \dot{d})$ by $V(\lambda, \dot{c})$. Generally, not all weight spaces of $V(\lambda, \dot{c}, \dot{d})$ are finite-dimensional. Recently, the necessary and sufficient conditions for $V(\lambda, \dot{c})$ to have finite-dimensional weight spaces were determined for $n=1$ in [RZ]. All the modules constructed in Sections 2 and 3 have finite-dimensional weight spaces. The following result is quite clear.

Theorem 5.1 (a) In Theorem 2.2, $B \simeq V(\lambda, 1,0)$, where $\lambda_{\alpha}=\frac{q^{-m \alpha}}{1-q^{\alpha}}$ for all $\alpha=\left(\alpha_{1}, \ldots, \alpha_{n}\right)$ $\in \mathbb{Z}^{n}$ with $q^{\alpha} \neq 1$, and $\lambda_{\alpha}=m$ otherwise. The highest weight vector in $B$ is 1 .

(b) In Theorem 3.6 (or 3.4), $S\left(\hat{H}_{I}^{-}\right) \simeq V(\lambda, m, 0)$, where

$$
\lambda_{\alpha}=\sum_{j=1}^{m} \frac{\omega_{m}^{-j \alpha_{1}}}{\left(1-q^{\alpha}\right)}=\delta_{\bar{\alpha}_{1}, 0} \frac{m}{\left(1-q^{\alpha}\right)}, \quad \forall \alpha=\left(\alpha_{1}, \ldots, \alpha_{n}\right) \in \mathbb{Z}^{n} \text { with } q^{\alpha} \neq 1
$$

and $\lambda_{\alpha}=0$ otherwise. The highest weight vector in $S\left(\hat{H}_{I}^{-}\right)$is 1 .

(c) In Theorem 4.4 (or 4.1), $V_{Q_{I}} \simeq V(\lambda, m, 0)$, where

$$
\lambda_{\alpha}=\delta_{\bar{\alpha}_{1}, 0} \sum_{j=1}^{m} \frac{\omega_{m}^{-j \alpha_{2}}}{\left(1-q^{\alpha}\right)}=\delta_{\bar{\alpha}_{1}, 0} \delta_{\bar{\alpha}_{2}, 0} \frac{m}{\left(1-q^{\alpha}\right)}, \quad \forall \alpha=\left(\alpha_{1}, \ldots, \alpha_{n}\right) \in \mathbb{Z}^{n} \text { with } q^{\alpha} \neq 1
$$

and $\lambda_{\alpha}=0$ otherwise. The highest weight vector in $V_{Q_{I}}$ is $1 \otimes 1$.

(d) In Theorem 4.6 (or 4.5), $\left(V_{Q_{I}}\right)^{r} \simeq V(\lambda, m r, 0)$, where

$$
\begin{aligned}
& \lambda_{\alpha}=\delta_{\bar{\alpha}_{1}, 0} \sum_{i=1}^{r} \omega_{r}^{-i \alpha_{l}} \sum_{j=1}^{m} \frac{\omega_{m}^{-j \alpha_{2}}}{\left(1-q^{\alpha}\right)} \\
& \quad=\delta_{\bar{\alpha}_{1}, 0} \delta_{\bar{\alpha}_{2}, 0} \delta_{\bar{\alpha}_{l}, 0} \frac{m r}{\left(1-q^{\alpha}\right)}, \quad \forall \alpha=\left(\alpha_{1}, \ldots, \alpha_{n}\right) \in \mathbb{Z}^{n} \text { with } q^{\alpha} \neq 1,
\end{aligned}
$$

and $\lambda_{\alpha}=0$ otherwise. The highest weight vector in $\left(V_{Q_{I}}\right)^{r}$ is $1=(1 \otimes 1)^{r}$.

We cannot ignore the fact that the character formulas in Theorems 3.2 and 4.1 are the same. This coincidence suggests that some isomorphism may exist. This is indeed the case. In Theorem 3.2 we know that $V_{Q_{I}}$ is an irreducible highest weight module with highest weight 
vector $1 \otimes 1$ by the following sense

$$
\left\{\begin{array}{l}
\left(E_{i, j}\left(t_{0}^{k} t^{a}\right)\right)(1 \otimes 1)=0, \quad \text { if } \quad k \in \mathbb{N}, \\
\left(E_{i, j}\left(t_{0}^{0} t^{a}\right)\right)(1 \otimes 1)=0, \quad \text { if } i \neq j, \\
c(1 \otimes 1)=1, \\
\left(E_{i, i}\left(t_{0}^{0} t^{a}\right)\right) 1 \otimes 1=\left\{\begin{array}{l}
\frac{1 \otimes 1}{\left(1-q^{\alpha}\right)}, \quad \text { if } q^{\alpha} \neq 1, \\
0, \quad \text { if } q^{\alpha}=1,
\end{array}\right.
\end{array}\right.
$$

for all $\alpha=\left(\alpha_{1}, \alpha_{2}, \ldots, \alpha_{n}\right) \in \mathbb{Z}$. Now we construct a new quantum torus $\mathbb{C}_{q^{\prime}}$ from $\mathbb{C}_{q}$ in Theorem 3.2 as follows: $\mathbb{C}_{q \prime}$ is generated by generators $t_{0}^{ \pm 1}, E^{ \pm 1}, F^{ \pm 1}, t_{1}^{ \pm 1}, \ldots t_{n}^{ \pm 1}$, where $t_{0}, t_{1}, \ldots t_{n}$ has the same relations as in $\mathbb{C}_{q}, E$ and $F$ commute with $t_{0}, t_{1}, \ldots t_{n}$, but $E F=\omega_{n} F E$. Homogeneous elements in $\mathbb{C}_{q^{\prime}}$ are written as $t_{0}^{\alpha_{0}} E^{\alpha_{1}} F^{\alpha_{2}} t_{1}^{\alpha_{3}} \ldots t_{n}^{\alpha_{n+2}}$ for $\alpha=\left(\alpha_{0}, \alpha_{1}, \alpha_{2}, \alpha_{3}, \ldots \alpha_{n+2}\right) \in \mathbb{Z}^{n+3}$. Then we have the irreducible highest weight $\widetilde{\mathbb{C}_{q^{\prime}}}$-module with highest weight vector $1 \otimes 1$ in Theorem 4.1 such that

$$
\left\{\begin{array}{l}
c(1 \otimes 1)=m, \quad\left(t_{0}^{\alpha_{0}} E^{\alpha_{1}} F^{\alpha_{2}} t_{1}^{\alpha_{3}} \ldots t_{n}^{\alpha_{n+2}}\right)(1 \otimes 1)=0, \quad \text { if } \alpha_{0} \in \mathbb{N}, \\
\left(t_{0}^{0} E^{\alpha_{1}} F^{\alpha_{2}} t_{1}^{\alpha_{3}} \ldots t_{n}^{\alpha_{n+2}}\right) 1 \otimes 1=\left\{\begin{array}{l}
\delta_{\bar{\alpha}_{1}, 0} \delta_{\bar{\alpha}_{2}, 0} \frac{m}{\left(1-q^{\alpha}\right)}, \quad \text { if } \quad q^{\alpha} \neq 1, \\
0, \quad \text { if } q^{\alpha}=1,
\end{array}\right.
\end{array}\right.
$$

where $q^{\alpha}=q_{1,0}^{\alpha_{3}} \ldots q_{n, 0}^{\alpha_{n+3}}$. Via the Lie homomorphism

$$
\left\{\begin{array}{l}
\tilde{g} l_{m}\left(\mathbb{C}_{q}\right) \rightarrow \widetilde{\mathbb{C}_{q \prime}} \\
c \rightarrow \frac{c}{m}, \quad d_{0} \rightarrow d_{0} \\
E_{i, j}\left(t_{0}^{k} t^{a}\right) \rightarrow \frac{1}{m} t_{0}^{k} E^{j-i}\left(\omega_{m}^{-j} F+\left(\omega_{m}^{-j} F\right)^{2}+\ldots+\left(\omega_{m}^{-j} F\right)^{m}\right) t_{1}^{\alpha_{1}} \ldots t_{n}^{\alpha_{n}}
\end{array}\right.
$$

one can verify that the modules defined in (5.3) and (5.4) are the same. Thus the module in Theorem 3.2 is a module in Theorem 4.1 with $q_{1,0}=q_{2,0}=1$. Thus we have proved

Theorem 5.2 The module in Theorem 3.2 is a module in Theorem 4.1 with $q_{1,0}=q_{2,0}=1$.

\section{References}

[BB] S. Berman, Y. Billig, Irreducible representations for toroidal Lie algebras, J. Algebra, 221(1999), no.1, 188-231.

[BGK] S. Berman, Y. Gao, Y.S. Krylyuk, Quantum tori and the structure of elliptic quasisimple Lie algebras, J. Funct. Anal., 135(1996), no.2, 339-389.

[BGT] S. Berman, Y. Gao, S. Tan, A unified view of some vertex operator constructions, Israel J. Math., to appear.

[BS] S. Berman, J. Szmigielski, Principal realization for the extended affine Lie algebra of type $\mathrm{sl}_{2}$ with coordinates in a simple quantum torus with two generators, Recent developments 
in quantum affine algebras and related topics (Raleigh, NC, 1998), 39-67, Contemp. Math., 248, Amer. Math. Soc., Providence, RI, 1999.

[DJKM] E. Date, M. Jimbo, M. Kashiwara, T. Miwa, Operator approach to the KP equation, Transformation groups for soliton equations. III, J. Phys. Soc. Japan, 50(1981), 38063812 .

[Fe] A.J. Feingold, Constructions of vertex operator algebras, Algebraic groups and their generalizations: quantum and infinite-dimensional methods (University Park, PA, 1991), 317-336, Proc. Sympos. Pure Math., 56, Part 2, Amer. Math. Soc., Providence, RI, 1994.

[F] I. B. Frenkel, Representations of affine Lie algebras, Hecke modular forms and Kortewegde Vries type equations, Lie algebras and related topics (New Brunswick, N.J., 1981), 71-110, Lecture Notes in Math., 933, Springer, Berlin-New York, 1982.

[G1] Y. Gao, Representations of extended affine Lie algebras coordinatized by certain quantum tori, Compositio Math., 123 (2000), no. 1, 1-25.

[G2] Y. Gao, Vertex operators arising from the homogeneous realization for $\widehat{\mathrm{gl}}_{N}$, Comm. Math. Phys., 211(2000), no.3, 745-777.

[GL] M. Golenishcheva-Kutuzova, D. Lebedev, Vertex operator representation of some quantum tori Lie algebras, Comm. Math. Phys., 148(1992), no.2, 403-416.

[KKLW] V.G. Kac, D.A. Kazhdan, J. Lepowsky, R.L. Wilson, Realization of the basic representations of the Euclidean Lie algebras, Adv. Math., 42(1981), no.1, 83-112.

[KP] V.G. Kac, D.H. Peterson, 112 constructions of the basic representations of loop groups of $E_{8}$, Proc. Symposium "Anomalies, geometry, Topology", World Wide Scientific, 1985, 276-298.

[KR] V.G. Kac and A.K. Raina, Bombay lectures on highest weight representations of infinite dimensional Lie algebras, World Sci., Singapore, 1987.

[LW] J. Lepowsky, R.L. Wilson, Construction of the affine Lie algebra $A_{1}^{(1)}$, Comm. Math. Phys., 62(1978), 43-53.

[MP] J. C. McConnell, J. J. Pettit, Crossed products and multiplicative analogues of Weyl algebras, J. London Math. Soc.(2)38, no.1, 47-55(1988).

[RZ] S. Eswara Rao, K. Zhao, Highest weight irreducible representations of quantum tori, preprint. 
[TV] F. ten Kroode, J. van de Leur, Bosonic and fermionic realizations of the affine algebra $\widehat{\mathrm{gl}}_{n}$, Comm. Math. Phys., 137(1991), no.1, 67-107.

[Z] K. Zhao, Weyl type algebras from quantum tori, preprint. 